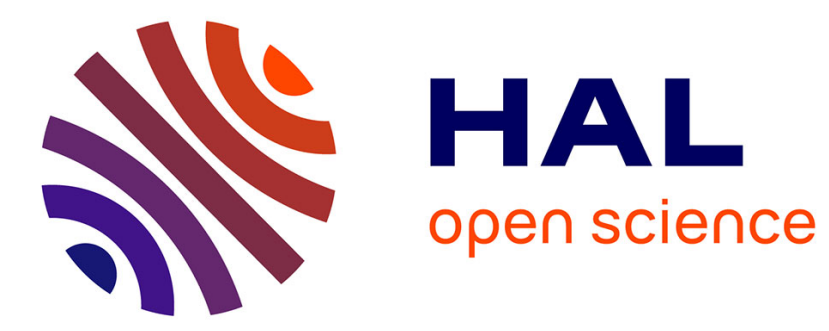

\title{
A model for interest rates with clustering effects
}

Donatien Hainaut

\section{To cite this version:}

Donatien Hainaut. A model for interest rates with clustering effects. Quantitative Finance, 2016, Vol. 16 (Issue 8), pp. 1203-1218. 10.1080/14697688.2015.1135251 . hal-01393994

\section{HAL Id: hal-01393994 \\ https://hal-rennes-sb.archives-ouvertes.fr/hal-01393994}

Submitted on 8 Nov 2016

HAL is a multi-disciplinary open access archive for the deposit and dissemination of scientific research documents, whether they are published or not. The documents may come from teaching and research institutions in France or abroad, or from public or private research centers.
L'archive ouverte pluridisciplinaire HAL, est destinée au dépôt et à la diffusion de documents scientifiques de niveau recherche, publiés ou non, émanant des établissements d'enseignement et de recherche français ou étrangers, des laboratoires publics ou privés. 


\title{
A model for interest rates with clustering effects
}

\author{
DONATIEN HAINAUT ${ }^{\dagger}$ \\ August 24, 2015 \\ $\dagger$ ESC Rennes Business School \& CREST, France. \\ Email: donatien.hainaut@esc-rennes.fr
}

\begin{abstract}
Abstract: We propose a model for short-term rates driven by a self-exciting jump process to reproduce the clustering of shocks on the Euro overnight index average (EONIA). The key element of the model is the feedback effect between the absolute value of jumps and the intensity of their arrival process. In this setting, we obtain a closed-form solution for the characteristic function for interest rates and their integral. We introduce a class of equivalent measures under which the features of the process are preserved. We infer the prices of bonds and their dynamics under a risk-neutral measure. The question of derivatives pricing is developed under a forward measure, and a numerical algorithm is proposed to evaluate caplets and floorlets. The model is fitted to EONIA rates from 2004 to 2014 using a peaksover-threshold procedure. From observation of swap curves over the same period, we filter the evolution of risk premiums for Brownian and jump components. Finally, we analyze the sensitivity of implied caplet volatility to parameters defining the level of self-excitation.
\end{abstract}

KEYWORDS. Hawkes process, self-exciting process, interest rates, yield curve.

\section{Introduction}

For most assets it has been observed that extreme events such as jumps in prices tend to occur in clusters (Ait-Sahalia et al., 2014a). Our analysis of the Euro overnight index average (EONIA) from 2004 to 2014 reveals that interbank interest rates exhibit similar behavior. However, the majority of continuous time models for interest rates are driven by Markov (Brownian and other Lévy) processes that cannot capture clustering effects because of the independence of their increments. The literature on these approaches is vast. The first interest rate models based on a single mean-reverting Brownian process were introduced by Vasicek (1977), Cox et al. (1985), and Hull and White (1990). Duffie and Kan (1996) and Dai and Singleton (2000) extended this framework to several risk factors and introduced affine models. Brigo and Mercurio (2007) provide a more complete survey. Interest rate models based on other Lévy processes are less popular, mainly because no closed-form solutions for options pricing exist. This type of model was explored by Eberlein and Kluge (2006), Filipović and Tappe (2008), and Hainaut and MacGilchrist (2010).

This paper contributes to the literature by proposing a different approach designed around a feedback mechanism to capture clustering effects in the evolution of interest rates. The feedback element introduces a propagation mechanism from one positive or negative shock on rates to the next, which is not present in common models. The main tools we use are Hawkes processes (Hawkes, 1971a,b; Hawkes and Oakes, 1974). These are parsimonious self-exciting point processes for which the intensity jumps in response to and reverts to a target level in the absence of an event. As the future of a self-exciting process is influenced by the timing of past events, Errais et al. (2010) used this to generate contagion between defaults in a top-down approach to credit risk. Embrechts et al. (2011) applied multivariate Hawkes processes in their analysis of stocks markets. Hawkes processes were also used by Ait-Sahalia et al. (2014a,b) to model 
two key aspects of asset prices: clustering in time and cross-sectional contamination between regions. These processes are increasingly integrated in high-frequency finance. Examples include modeling of the duration between trades (Bauwens and Hautsch, 2009) and of the arrival process for buy and sell orders (Bacry et al., 2013). Giot (2005), Chavez-Demoulin et al. (2005), and Chavez-Demoulin and McGill (2012) tested these processes in a risk management context, whereas Dassios and Jang (2012) proposed a bivariate process for applications in insurance.

This study complements the existing literature in several directions. It is one of the first studies to use self-exciting processes to model the term structure of interest rates. The papers cited above focus on stocks or CDS markets. In most econometric applications published, jumps are unidirectional and constant to preserve the positivity of the intensity of the Poisson process. Our EONIA study emphasizes that these assumptions are not relevant for interbank interest rate markets. In these markets, jumps have a variable amplitude and are caused by successive adjustments, whether positive or negative, of rates for deposit and marginal lending facilities offered by central banks. To include this feature in our model, the intensity of shocks is not linked to the aggregate value of all past jumps, but to the sum of their absolute values. This approach may be viewed as a parsimonious alternative to the bivariate Hawkes processes used by Embrechts et al. (2011). In addition, our work provides all the tools for pricing bonds and reconciling the dynamics of the short-term rate under a real measure, with the term structure of bonds yields evaluated under a risk-neutral measure. In particular, we propose a family of changes of measures (risk-neutral and forward) that preserves the dynamics of the process under real and risk-neutral measures. Finally, after describing the dynamics of bonds quotes, we present the moment-generating function of yields under a forward measure and use this in numerical applications for the pricing of derivatives.

To justify the presence of a self-exciting component in the dynamics of short-term rates, we fit our model to an EONIA time series over a period of ten years. This index is a weighted average of all overnight unsecured lending transactions (in euros) between prime banks. As the sample paths for jumps and their intensities are not observable, calibration cannot be performed via direct log likelihood maximization. To solve this inference problem, Ait-Sahalia et al. (2014a) applied the generalized method of moments (GMM) to stock returns. Ait-Sahalia et al. (2014b) used an alternative method based on matching modeled and market prices. Instead, we opt for a peak-over-threshold procedure, as used by Embrechts et al. (2011). This method allows us to filter jumps and the intensity of their arrival process. Parameters are next inferred via three independent log likelihood maximization procedures. To explain the evolution of swap curves over the same period, we adjust daily risk premiums by matching modeled and market swap rates. Finally, we analyze the sensitivity of caplet implied volatility to parameters defining the level of self-excitation.

\section{$2 \quad$ Interest rate model}

Hawkes processes belong to the family of point processes and are used in seismology to model the frequency of earthquakes and clustering of aftershocks. The intensity of the arrival of events directly depends on the history of the process and increases after the occurrence of a jump. This propagation mechanism has been adapted in finance research to capture clustering effects. Except for work by Salmon and Tham (2007), very few papers have investigated this phenomenon in interest rate markets. Nevertheless, similar trends are observed for EONIA rates, as emphasized by Figure 2, which shows rate jumps from 1/1/2004 to 31/12/2014 after filtering via a procedure described in detail later. Most of these jumps are caused by successive adjustments of rates for deposit and marginal lending facilities offered by central banks. The clustering of the adjustments 
is explained by the emergency of such decisions in periods of economic crisis. This observation is at the heart of our study. We propose an interest rate model that duplicates the clustering of shocks and explains the term structure of bond yields. To achieve this goal, the dynamics of the short-term rate is driven by the sum of a mean-reverting function, a Brownian process, and a Hawkes process. This short-term interest rate is denoted by $r_{t}$ and is assumed to be defined by the stochastic differential equation (SDE)

$$
d r_{t}=a\left(\theta(t)-r_{t}\right) d t+\sigma d W_{t}+d\left(\sum_{i=1}^{N_{t}} J_{i}\right)
$$

on a complete probability space $(\Omega, \mathcal{F}, P)$ with right-continuous information filter $\mathbb{F}=\left(\mathcal{F}_{t}\right)_{t>0}$, where $P$ denotes the real probability measure. $\theta(t)>0$ is the mean level to which interest rates tend to revert and $a$ is the speed of mean reversion. $W_{t}$ is Brownian motion and $\sigma$ is Brownian volatility. $N_{t}$ is a Poisson process with intensity $\lambda_{t}$, and $J_{i}$ are i.i.d. random jumps with density $\nu(z)$ on $\mathbb{R}$. Most of results developed in the next sections are independent of the distribution chosen for jumps. However, we choose to work with double-exponential jumps in numerical applications. The density in this case is defined by the three parameters $\rho^{+} \in \mathbb{R}^{+}, \rho^{-} \in \mathbb{R}^{-}$and $p \in(0,1)$ according to

$$
\nu(z)=p \rho^{+} e^{-\rho^{+} z} 1_{\{z \geq 0\}}-(1-p) \rho^{-} e^{-\rho^{-} z} 1_{\{z<0\}},
$$

and the cumulative distribution function is given by

$$
P\left(J_{i} \leq z\right)=\left\{\begin{array}{ll}
(1-p) e^{-\rho^{-} z} & z \leq 0 \\
(1-p)+p\left(1-e^{-\rho^{+} z}\right) & z>0
\end{array} .\right.
$$

In this distribution, $p$ and $(1-p)$ are the probability of observing upward and downward exponential jumps, respectively, and the expectation of $J$ is equal to a weighted sum of expected average jumps:

$$
\mathbb{E}\left(J_{i}\right)=p \frac{1}{\rho^{+}}+(1-p) \frac{1}{\rho^{-}} .
$$

In following developments, we need the moment-generating function for the sum of $J$ and of its absolute value, given by

$$
\begin{aligned}
\psi\left(z_{1}, z_{2}\right) & :=\mathbb{E}\left(e^{z_{1} J_{i}+z_{2}\left|J_{i}\right|}\right) \\
& :=p \frac{\rho^{+}}{\rho^{+}-\left(z_{1}+z_{2}\right)}+(1-p) \frac{\rho^{-}}{\rho^{-}-\left(z_{1}-z_{2}\right)}
\end{aligned}
$$

if $\left(z_{1}+z_{2}\right)<\rho^{+}$and $\left(z_{1}-z_{2}\right)>\rho^{-}$. The proof is provided in Appendix A. The dynamics chosen for $r_{t}$ allows negative interest rates but we do not consider this as a limitation. Indeed, since the European sovereign debt crisis in 2012, we have observed several periods during which short-term rates (sovereign or interbank) were negative (e.g. in 2014, the EONIA was negative 61 times during 254 days of trading). Propagation is modeled by assuming that the jump arrival frequency $\lambda_{t}$ depends on the sum of absolute values for jumps up to time $t$, denoted by $L_{t}$ :

$$
L_{t}=\sum_{i=1}^{N_{t}}\left|J_{i}\right| d N_{t} .
$$

The frequency $\lambda_{t}$ is driven by

$$
d \lambda_{t}=\kappa\left(c-\lambda_{t}\right) d t+\delta d L_{t}
$$


where $\delta, \kappa$, and $c$ are positive and constant. By construction, the feedback process for jump frequency is strictly positive and ensures the positivity of $\lambda_{t}$. As mentioned in the Introduction, jumps are unidirectional (exclusively positive or negative) in most previous studies. In our approach, jumps can be either positive or negative, but any jump occurrence increases the intensity of $N_{t}$. Even if this feature seems anecdotal, it introduces some additional difficulty, particularly in guessing the dynamics of jumps under a risk-neutral or forward measure. This point is further discussed later. However, we can first check via direct differentiation that the solution of Eq. (5) is given by

$$
\lambda_{t}=c+e^{-\kappa t}\left(\lambda_{0}-c\right)+\delta \int_{0}^{t} e^{-\kappa(t-s)} d L_{s}
$$

The expectation for $\lambda_{t}$ is provided by the next proposition. This result was demonstrated by Errais et al. (2010) but an alternative proof is presented here.

Proposition 2.1. The expected value of $\lambda_{t}$ is equal to

$$
\mathbb{E}\left(\lambda_{t} \mid \mathcal{F}_{0}\right)=\left(\frac{\kappa c}{\delta \mathbb{E}\left(\left|J_{i}\right|\right)-\kappa}+\lambda_{0}\right) e^{\left(\delta \mathbb{E}\left(\left|J_{i}\right|\right)-\kappa\right) t}-\frac{\kappa c}{\delta \mathbb{E}\left(\left|J_{i}\right|\right)-\kappa} .
$$

Proof. As jumps are independent of the intensity, we infer from Eq. (6) that

$$
\begin{aligned}
\mathbb{E}\left(\lambda_{t} \mid \mathcal{F}_{0}\right) & =c+e^{-\kappa t}\left(\lambda_{0}-c\right)+\delta \int_{0}^{t} e^{-\kappa(t-s)} \mathbb{E}\left(d L_{s} \mid \mathcal{F}_{0}\right) \\
& =c+e^{-\kappa t}\left(\lambda_{0}-c\right)+\delta \int_{0}^{t} e^{-\kappa(t-s)} \mathbb{E}\left(\left|J_{i}\right|\right) \mathbb{E}\left(\lambda_{s-} \mid \mathcal{F}_{0}\right) d s
\end{aligned}
$$

If we take the derivative of the last expression with respect to time, we find that $\mathbb{E}\left(\lambda_{t} \mid \mathcal{F}_{0}\right)$ is the solution of the ordinary differential equation (ODE)

$$
\begin{aligned}
\frac{\partial}{\partial t} \mathbb{E}\left(\lambda_{t} \mid \mathcal{F}_{0}\right) & =-\kappa e^{-\kappa t}\left(\lambda_{0}-c\right)+\delta \mathbb{E}\left(\left|J_{i}\right|\right) \mathbb{E}\left(\lambda_{t} \mid \mathcal{F}_{0}\right)-\kappa \delta \int_{0}^{t} e^{-\kappa(t-s)} \mathbb{E}\left(\left|J_{i}\right|\right) \mathbb{E}\left(\lambda_{s-} \mid \mathcal{F}_{0}\right) d s \\
& =\left(\delta \mathbb{E}\left(\left|J_{i}\right|\right)-\kappa\right) \mathbb{E}\left(\lambda_{t} \mid \mathcal{F}_{0}\right)+\kappa c
\end{aligned}
$$

which admits Eq. (7) as a solution.

From Eq. (7), we deduce the following parameter condition defining the dynamics of $\lambda_{t}$

$$
\delta \mathbb{E}\left(\left|J_{i}\right|\right)<\kappa
$$

to ensure the asymptotic stability of the process $\left(\lim _{t \rightarrow \infty} \mathbb{E}\left(\lambda_{t} \mid \mathcal{F}_{0}\right)<\infty\right)$. If this condition is fulfilled, the intensity converges asymptotically towards $\frac{\kappa c}{\kappa-\delta \mathbb{E}\left(\left|J_{i}\right|\right)}$. When jumps are doubleexponential random variables, the expectation for their absolute value is $\mathbb{E}\left(\left|J_{i}\right|\right)=p \frac{1}{\rho^{+}}+(1-$ p) $\frac{1}{\left|\rho^{-}\right|}$and inequality (9) is easy to check.

Having defined the dynamics of the short-term rate, we now present its infinitesimal generator. If $I_{t}=\left(N_{t}, L_{t}\right)$, the process $\left(r_{t}, \lambda_{t}, I_{t}\right)$ is a Markov process in the state space $D=$ $\left(\mathbb{R} \times \mathbb{R}^{+} \times \mathbb{N} \times \mathbb{R}^{+}\right)$and its infinitesimal generator for any function $g\left(t, r_{t}, \lambda_{t}, I_{t}\right): \mathbb{R}^{+} \times D \rightarrow \mathbb{R}$ with partial derivatives $g_{t}, g_{\lambda}, g_{r}$, and $g_{r r}$ is defined by

$$
\begin{aligned}
\mathcal{A} g\left(t, r_{t}, \lambda_{t}, I_{t}\right) & =g_{t}+a\left(\theta-r_{t}\right) g_{r}+\frac{1}{2} \sigma^{2} g_{r r}+\kappa\left(c-\lambda_{t}\right) g_{\lambda} \\
& +\lambda_{t} \int_{-\infty}^{-\infty} g\left(t, r_{t}+z, \lambda_{t}+\delta|z|, I_{t}+(1,|z|)^{\top}\right)-g\left(t, r_{t}, \lambda_{t}, I_{t}\right) \nu(d z) .
\end{aligned}
$$


Under mild conditions, the expectation for $g($.$) is equal to the integral of the expected infinites-$ imal generator:

$$
\begin{aligned}
\mathbb{E}\left(g\left(T, r_{T}, \lambda_{T}, I_{T}\right) \mid \mathcal{F}_{t}\right) & =g\left(t, r_{t}, \lambda_{t}, I_{t}\right)+\mathbb{E}\left(\int_{t}^{T} \mathcal{A} g\left(s, r_{s}, \lambda_{s}, I_{s}\right) d s \mid \mathcal{F}_{t}\right) \\
& =g\left(t, r_{t}, \lambda_{t}, I_{t}\right)+\int_{t}^{T} \mathbb{E}\left(\mathcal{A} g\left(s, r_{s}, \lambda_{s}, I_{s}\right) \mid \mathcal{F}_{t}\right) d s .
\end{aligned}
$$

The derivative of this expectation with respect to time is equal to its expected infinitesimal generator:

$$
\frac{\partial}{\partial T} \mathbb{E}\left(g\left(T, r_{T}, \lambda_{T}, I_{T}\right) \mid \mathcal{F}_{t}\right)=\mathbb{E}\left(\mathcal{A} g\left(T, r_{T}, \lambda_{T}, I_{T}\right) \mid \mathcal{F}_{t}\right)
$$

The next proposition shows that the moment-generating function for $\left(r_{T}, \lambda_{T},-\int_{t}^{T} r_{s} d s\right)$ is an affine function of the short-term rate and of the intensity. This result is used later to price bonds under a risk-neutral measure.

Proposition 2.2. If we note that $\psi\left(z_{1}, z_{2}\right):=\mathbb{E}\left(e^{z_{1} J_{i}+z_{2}\left|J_{i}\right|}\right)$, the moment-generating function for $\left(r_{T}, \lambda_{T},-\int_{t}^{T} r_{s} d s\right)$ is an affine function of $r_{t}$ and $\lambda_{t}$ :

$$
\mathbb{E}\left(e^{w_{0} r_{T}+w_{1} \lambda_{T}-w_{2} \int_{t}^{T} r_{s} d s} \mid \mathcal{F}_{t}\right)=\exp \left(A(t, T)+B(t, T) r_{t}+C(t, T) \lambda_{t}\right),
$$

where $A(t, T), B(t, T)$, and $C(t, T)$ are solutions of the ODE system

$$
\left\{\begin{array}{l}
\frac{\partial}{\partial t} A(t, T)=-a \theta(t) B-\kappa c C-\frac{1}{2} \sigma^{2} B^{2} \\
\frac{\partial}{\partial t} B(t, T)=a B+w_{2} \\
\frac{\partial}{\partial t} C(t, T)=\kappa C-[\psi(B, C \delta)-1]
\end{array}\right.
$$

and satisfy the terminal conditions $A(T, T)=0, B(T, T)=w_{0}$, and $C(T, T)=w_{1}$.

Proof. We define $Y_{t}:=\mathbb{E}\left(e^{w_{0} r_{T}+w_{1} \lambda_{T}-w_{2} \int_{t}^{T} r_{s} d s} \mid \mathcal{F}_{t}\right)$. As $\mathcal{F}_{t} \subset \mathcal{F}_{u}$ for any $u \geq t$, applying the rule of conditional expectations leads to

$$
\begin{aligned}
Y_{t} & =\mathbb{E}\left(e^{-w_{2} \int_{t}^{u} r_{s} d s} \mathbb{E}\left(e^{w_{0} r_{T}+w_{1} \lambda_{T}-w_{2} \int_{u}^{T} r_{s} d s} \mid \mathcal{F}_{u}\right) \mid \mathcal{F}_{t}\right) \\
& =\mathbb{E}\left(e^{w_{0} r_{T}+w_{1} \lambda_{T}-w_{2} \int_{t}^{u} r_{s} d s} Y_{u} \mid \mathcal{F}_{t}\right) .
\end{aligned}
$$

Then, assuming enough regularity to allow us to take the limit within the expectation, the following limit converges to zero:

$$
\lim _{u \rightarrow t} \frac{\mathbb{E}\left(e^{-w_{2} \int_{t}^{u} r_{s} d s} Y_{u} \mid \mathcal{F}_{t}\right)-Y_{t}}{u-t}=0 .
$$

If we develop the exponential as its Taylor approximation of first order, the limit is rewritten as

$$
\lim _{u \rightarrow t} \frac{\mathbb{E}\left(Y_{u} \mid \mathcal{F}_{t}\right)-Y_{t}}{u-t}=w_{2} r_{t} Y_{t}
$$

in which the left-hand term is the infinitesimal generator of the moment-generating function. To simplify future calculations, we let $f\left(t, r_{t}, \lambda_{t}, I_{t}\right):=Y_{t}$ and denote $f_{t}, f_{\lambda}$, and $f_{r}, f_{r r}$ as the partial derivatives of $f$ with respect to time, intensity and interest rate. Then Eq. (16) is equal to $\mathcal{A} f=w_{2} r_{t} f$, and after development to

$$
\begin{aligned}
& w_{2} r_{t} f=f_{t}+a\left(\theta(t)-r_{t}\right) f_{r}+\kappa\left(c-\lambda_{t}\right) f_{\lambda}+\frac{1}{2} \sigma^{2} f_{r r} \\
& +\lambda_{t} \int_{-\infty}^{+\infty} f\left(t, r_{t}+z, \lambda_{t}+\delta|z|, I_{t}+(1,|z|)^{\top}\right)-f \nu(d z),
\end{aligned}
$$


and $f$ satisfies the terminal condition

$$
f\left(T, r_{T}, \lambda_{T}, I_{T}\right)=\exp \left(w_{0} r_{T}+w_{1} \lambda_{T}\right) .
$$

In the remainder of this proof, we assume that $f$ is an exponential affine function of $r_{t}$ and $\lambda_{t}$ :

$$
f=\exp \left(A(t, T)+B(t, T) r_{t}+C(t, T) \lambda_{t}\right)
$$

where $A(t, T), B(t, T)$, and $C(t, T)$ are functions of time. Under this assumption, the partial derivatives of $f$ are given by

$$
\begin{aligned}
& f_{t}=\left(\frac{\partial}{\partial t} A+r_{t} \frac{\partial}{\partial t} B+\lambda_{t} \frac{\partial}{\partial t} C\right) f \\
& f_{r}=B f \quad f_{r r}=B^{2} f \quad f_{\lambda}=C f,
\end{aligned}
$$

and the integrand in Eq. (17) can be rewritten as

$$
f\left(t, r_{t}+z, \lambda_{t}+\delta|z|, I_{t}+(1,|z|)^{\top}\right)-f=f[\exp (B z+C \delta|z|)-1] .
$$

Developing Eq. (17) leads to the relation

$$
\begin{aligned}
& 0=\frac{\partial}{\partial t} A+a \theta B+\kappa c C+\frac{1}{2} \sigma^{2} B^{2}+r_{t}\left(\frac{\partial}{\partial t} B-a B-w_{2}\right) \\
& +\lambda_{t}\left(\frac{\partial}{\partial t} C-\kappa C+[\psi(B, C \delta)-1]\right) .
\end{aligned}
$$

As $r_{t}$ and $\lambda_{t}$ are random variables, this last relation holds only if their multiplicative coefficients are null. This is achieved if $A(t, T), B(t, T)$, and $C(t, T)$ satisfy system (14).

In fact, it is easy to check that $B(t, T)$ admits the following closed-form expression:

$$
B(t, T)=\frac{1}{a}\left(-w_{2}+\left(w_{2}+a w_{0}\right) e^{-a(T-t)}\right) .
$$

In numerical applications, the functions $A(t, T)$ and $C(t, T)$ are computed using the Euler method. Note that it is also possible to numerically retrieve the probability density function (pdf) for $r_{t}$ by numerically inverting its moment-generating function using a fast Fourier algorithm. However, we see later that model calibration against historical time series via log likelihood is not necessary. Instead, we use a peak-over-threshold procedure to detect jumps and fit the jump and Brownian processes separately. This procedure is described in Section 4.

\section{Equivalent exponential affine measures and bond pricing}

A fundamental step in identifying at least one class of equivalent measures of probability is definition of a risk-neutral measure. This step is required for subsequent reconciliation of the econometric calibration results obtained under a historical measure with the term structure for bond yields evaluated in a risk-neutral world. This also allows us to measure the risk premiums related to the Brownian and jump components embedded in the dynamics of $r_{t}$. However, as there is no contingent claim written for $\lambda_{t}$, the market is incomplete and there are an infinite number of eligible equivalent risk-neutral measures. There is also no guarantee that the dynamics of $r_{t}$ is similar in the real and risk-neutral worlds. For this reason, we focus on a family of exponential 
affine changes of measure and find the conditions under which the interest rate dynamics is preserved. These equivalent measures are induced by exponential martingales of the form

$$
M_{t}(\gamma, \xi):=\exp \left(g(\gamma) \lambda_{t}+\gamma L_{t}-\varphi(\gamma) t-\frac{1}{2} \int_{0}^{t} \xi^{2} d s-\int_{0}^{t} \xi d W_{s}\right),
$$

where $\xi$ defines the market price for interest risk. Zhang et al. (2009) used a similar change of measure to simulate rare events of a one-dimension Hawkes process without a Brownian component and with only constant jumps. In our framework, jumps are random and the affine change of measure modifies both the frequency and distribution of jumps. Before detailing this point, the next proposition introduces the conditions that $\gamma$ must fulfill to guarantee that $M_{t}(\gamma)$ is a local martingale.

Proposition 3.1. If for $\gamma$ there exists a suitable solution $g(\gamma)$ for the equation

$$
g \kappa-(\psi(0, \gamma+g \delta)-1)=0
$$

where $\psi(0, z)=\mathbb{E}\left(e^{z\left|J_{i}\right|}\right)$, and if $\varphi(\gamma)$ is defined by

$$
\varphi(\gamma)=g(\gamma) \kappa c
$$

then $M_{t}(\gamma)$ is a local martingale.

Proof. We denote by $Y_{t}$ the exponent of $M_{t}$ defined by Eq. (19):

$$
Y_{t}=g(\gamma) \lambda_{t}+\gamma L_{t}-\varphi(\gamma) t-\frac{1}{2} \int_{0}^{t} \xi^{2} d s-\int_{0}^{t} \xi d W_{s}
$$

According to Eq. (5), the infinitesimal dynamics is given by

$$
d Y_{t}=g \kappa\left(c-\lambda_{t}\right) d t+(g \delta+\gamma) d L_{t}-\varphi(\gamma) d t-\frac{1}{2} \xi^{2} d t-\xi d W_{t}
$$

In the remainder of this proof, the random measure of $J_{i}$ is denoted by $\chi($.$) and is such that$ $J_{i}=\int_{-\infty}^{\infty} \chi(d z)$. Applying the Itô lemma for semi-martingales to $M_{t}$ leads to the relation

$$
\begin{aligned}
& d M_{t}=M_{t} d Y_{t}+\frac{1}{2} M_{t} d\left[Y_{t}, Y_{t}\right]_{t}^{c} \\
& \quad+M_{t} \int_{-\infty}^{\infty}\left(e^{(g \delta+\gamma)|z|}-1-(g \delta+\gamma)|z|\right) \chi(d z) d N_{t}
\end{aligned}
$$

and this equation can be developed as follows:

$$
\begin{aligned}
d M_{t}= & M_{t}(g \kappa c-\varphi) d t-M_{t} \xi d W_{t}-\frac{1}{2} \xi^{2} M_{t} \\
& +\frac{1}{2} \xi^{2} M_{t}-M_{t} \lambda_{t}\left(g \kappa-\int_{-\infty}^{\infty}\left(e^{(g \delta+\gamma)|z|}-1\right) \nu(d z)\right) d t \\
& +M_{t} \int_{-\infty}^{\infty}\left(e^{(g \delta+\gamma)|z|}-1\right)\left[\chi(d z) d N_{t}-\lambda_{t} \nu(d z) d t\right]
\end{aligned}
$$

Since the integral with respect to $\chi(d z) d N_{t}-\lambda \nu(d z) d t$ is a local martingale, $M_{t}$ is also a local martingale if and only if the following relations hold:

$$
\begin{cases}g \kappa c-\varphi & =0 \\ g \kappa-\int_{-\infty}^{\infty}\left(e^{(g \delta+\gamma)|z|}-1\right) \nu(d z) & =0 .\end{cases}
$$


Assuming the existence of suitable solutions for Eqs. (20) and (21), an equivalent measure $Q^{\gamma, \xi}$ is defined by the Radon-Nykodym derivative

$$
\left.\frac{d Q^{\gamma, \xi}}{d P}\right|_{\mathcal{F}_{t}}=\frac{M_{t}(\gamma, \xi)}{M_{0}(\gamma, \xi)}
$$

and may be used as a risk-neutral measure. In this case, the dynamics of the short-term rate and of the intensity is modified but is still a combination of a mean-reverting Brownian process and a Hawkes process, as proved in the next proposition.

Proposition 3.2. Under the equivalent measure $Q^{\gamma, \xi}$, the short-term rate is still a meanreverting jump diffusion model driven by the dynamics

$$
d r_{t}=a\left(\theta^{Q}(t)-r_{t}\right) d t+\sigma d W_{t}^{Q}+d\left(\sum_{i=1}^{N_{t}^{Q}} J_{i}^{Q}\right),
$$

where the long-term trend to which interest rates revert is modified as follows:

$$
\theta^{Q}(t)=\theta(t)-\frac{\xi \sigma}{a} .
$$

Furthermore, $N_{t}^{Q}$ is a counting process with intensity $\lambda_{t}^{Q}=\psi(0, \delta g+\gamma) \lambda_{t}$ driven by the dynamics

$$
d \lambda_{t}^{Q}=\kappa\left(c^{Q}-\lambda_{t}^{Q}\right) d t+\delta^{Q} d L_{t}^{Q}
$$

where

$$
c^{Q}=\psi(0, \delta g+\gamma) c \quad \delta^{Q}=\psi(0, \delta g+\gamma) \delta .
$$

$J^{Q}$ denotes a random variable with the following characteristic function:

$$
\psi^{Q}\left(z_{1}, z_{2}\right):=\mathbb{E}^{Q}\left(e^{z_{1} J_{i}^{Q}+z_{2}\left|J_{i}^{Q}\right|}\right)=\frac{\psi\left(z_{1}, z_{2}+(\delta g+\gamma)\right)}{\psi(0, \delta g+\gamma)}
$$

and $L_{t}^{Q}$ is defined by the sum of jumps under $Q$ :

$$
L_{t}^{Q}=\sum_{i=1}^{N_{t}^{Q}}\left|J_{i}^{Q}\right| .
$$

Proof. If $Y_{t}$ is the exponent of $M_{t}$, as defined by Eq. (22), the characteristic function for $r_{T}$ under the risk-neutral measure is given by

$$
\mathbb{E}^{Q}\left(e^{w r_{T}} \mid \mathcal{F}_{t}\right)=e^{-Y_{t}} \mathbb{E}\left(e^{Y_{T}+w r_{T}} \mid \mathcal{F}_{t}\right)
$$

If $f\left(t, r_{t}, Y_{t}, \lambda_{t}, I_{t}\right)$ denotes $\mathbb{E}\left(e^{Y_{T}+w r_{T}} \mid \mathcal{F}_{t}\right)$, according to the Itô lemma, it solves the equation

$$
\begin{aligned}
& 0=f_{t}+a\left(\theta-r_{t}\right) f_{r}+\frac{1}{2} \sigma^{2} f_{r r}+\kappa\left(c-\lambda_{t}\right) f_{\lambda}-\xi \sigma f_{y r} \\
& +\left(g \kappa\left(c-\lambda_{t}\right)-\varphi(\gamma)-\frac{1}{2} \xi^{2}\right) f_{y}+\frac{1}{2} \xi^{2} f_{y y} \\
& +\lambda_{t} \int_{-\infty}^{+\infty} f\left(t, r_{t}+z, y_{t}+(g \delta+\gamma)|z|, \lambda+\delta|z|, I_{t}+(1,|z|)^{\top}\right)-f d \nu(z),
\end{aligned}
$$

where $f_{t}, f_{y}, f_{y y}$, and $f_{r}, f_{r r}$ are the partial derivatives of $f($.$) with respect to time and other$ state variables. As in the proof of Proposition 2.2, we assume that $f($.$) is an exponential affine$ function of risk factors:

$$
f=\exp \left(A(t, T)+B(t, T) r_{t}+C(t, T) \psi(0, \delta g+\gamma) \lambda_{t}+D(t, T) Y_{t}\right) .
$$


Under this assumption, the partial derivatives of $f$ are

$$
\begin{gathered}
f_{t}=\left(\frac{\partial}{\partial t} A+r_{t} \frac{\partial}{\partial t} B+\lambda_{t} \psi(0, \delta g+\gamma) \frac{\partial}{\partial t} C+Y_{t} \frac{\partial}{\partial t} D\right) f \\
f_{r}=B f \quad f_{r r}=B^{2} f \quad f_{\lambda}=C \psi(0, \delta g+\gamma) f \\
f_{y}=D f \quad f_{y y}=D^{2} f \quad f_{y r}=B D f
\end{gathered}
$$

and the integrand in Eq. (26) is rewritten as

$$
\begin{aligned}
f(t & \left., r_{t}+z, y_{t}+(g \delta+\gamma)|z|, \lambda+\delta|z|, I_{t}+(1,|z|)^{\top}\right) \\
& =f \exp (B z+(C \psi(0, \delta g+\gamma) \delta+D(\delta g+\gamma))|z|) .
\end{aligned}
$$

Inserting these expressions in Eq. (26) and canceling terms multiplying the state variables yields the ODE system

$$
\begin{aligned}
0= & \frac{\partial}{\partial t} A+a \theta B+\frac{1}{2} \sigma^{2} B^{2}+\kappa c C \psi(0, \delta g+\gamma) \\
& +\left(g \kappa c-\varphi(\gamma)-\frac{1}{2} \xi^{2}(1-D)-\xi \sigma B\right) D \\
0= & \frac{\partial}{\partial t} B-a B \\
0= & \psi(0, \delta g+\gamma) \frac{\partial}{\partial t} C-\kappa C \psi(0, \delta g+\gamma)-g \kappa D \\
0= & \frac{\partial}{\partial t} D \quad+[\psi(B, C \psi(0, \delta g+\gamma) \delta+D(\delta g+\gamma))-1]
\end{aligned}
$$

with the terminal conditions $A(T, T, w)=0, B(T, T, w)=w, C(T, T, w)=0$, and $D(T, T, w)=$ 1. From the last relation, we infer that $D=1$. As $\varphi(\gamma)=g(\gamma) \kappa c$ and $g \kappa=(\psi(0, g \delta+\gamma)-1)$, this last ODE system is finally rewritten as

$$
\begin{aligned}
0 & =\frac{\partial}{\partial t} A+a \theta^{Q} B+\frac{1}{2} \sigma^{2} B^{2}+\kappa c^{Q} C \\
0 & =\frac{\partial}{\partial t} B-a B \\
0 & =\frac{\partial}{\partial t} C-\kappa C+\left[\frac{\psi\left(B, C \delta^{Q}+(\delta g+\gamma)\right)}{\psi(0, \delta g+\gamma)}-1\right] .
\end{aligned}
$$

This completes the proof.

The next proposition shows that the jump distribution is preserved under $Q$.

Proposition 3.3. Under $Q$, jumps, $J_{i}^{Q}$ are double-exponential random variables with density

$$
\nu^{Q}(z)=p^{Q} \rho^{+Q} e^{-\rho^{+Q} z} 1_{\{z \geq 0\}}-\left(1-p^{Q}\right) \rho^{-Q} e^{-\rho^{-Q} z} 1_{\{z<0\}},
$$

where the parameters are adjusted as follows:

$$
\begin{aligned}
\rho^{+Q} & =\rho^{+}-(\delta g+\gamma) \\
\rho^{-Q} & =\rho^{-}+(\delta g+\gamma) \\
p^{Q} & =\frac{p \rho^{+} \rho^{-Q}}{\left(p \rho^{+} \rho^{-Q}+(1-p) \rho^{-} \rho^{+Q}\right)}
\end{aligned}
$$


Proof. By construction, the moment-generating function for jumps under the risk-neutral measure is the ratio

$$
\psi^{Q}\left(z_{1}, 0\right)=\frac{\psi\left(z_{1}, 0+(\delta g+\gamma)\right)}{\psi(0, \delta g+\gamma)} .
$$

If we denote $\alpha=(\delta g+\gamma)$, the numerator and denominator in this equation are given by

$$
\begin{aligned}
\psi\left(z_{1}, \alpha\right) & =\frac{p \rho^{+}\left(\rho^{-}+\alpha-z_{1}\right)+(1-p) \rho^{-}\left(\rho^{+}-\alpha-z_{1}\right)}{\left(\rho^{+}-\alpha-z_{1}\right)\left(\rho^{-}+\alpha-z_{1}\right)} \\
\psi(0, \alpha) & =\frac{p \rho^{+}\left(\rho^{-}+\alpha\right)+(1-p) \rho^{-}\left(\rho^{+}-\alpha\right)}{\left(\rho^{+}-\alpha\right)\left(\rho^{-}+\alpha\right)} .
\end{aligned}
$$

Then, since

$$
\psi^{Q}\left(z_{1}, 0\right)=\frac{\frac{p \rho^{+} \rho^{-Q}}{\left(p \rho^{+} \rho^{-Q}+(1-p) \rho^{-} \rho^{+Q}\right)}\left(\rho^{-Q}-z_{1}\right) \rho^{+Q}+\frac{(1-p) \rho^{-} \rho^{+Q}}{\left(p \rho^{+} \rho^{-Q}+(1-p) \rho^{-} \rho^{+Q}\right)} \rho^{-Q}\left(\rho^{+Q}-z_{1}\right)}{\left(\rho^{+Q}-z_{1}\right)\left(\rho^{-Q}-z_{1}\right)},
$$

the proof is complete.

The remainder of this section develops useful corollaries for the pricing of bonds. If market participants adopt an equivalent exponential affine measure for the risk-neutral measure, the price of a zero-coupon bond is equal to the expected discount factor under this measure. Hereafter, the price a bond at time $t$ and expiring at $T$ is denoted by

$$
P\left(t, T, r_{t}, \lambda_{t}^{Q}, I_{t}^{Q}\right)=\mathbb{E}^{Q}\left(e^{-\int_{t}^{T} r_{s} d s} \mid \mathcal{F}_{t}\right)
$$

From Proposition 2.2, it is easy to check that this price is also an affine function of short-term rates and intensities because the dynamics of $r_{t}$ under the real and risk-neutral measures is similar.

\section{Corollary 3.4.}

$$
\mathbb{E}^{Q}\left(e^{-\int_{t}^{T} r_{s} d s} \mid \mathcal{F}_{t}\right)=\exp \left(A^{P}(t, T)+B^{P}(t, T) r_{t}+C^{P}(t, T) \lambda_{t}^{Q}\right),
$$

where $A^{P}(t, T)$ and $C^{P}(t, T)$ are solutions of the ODE system

$$
\begin{aligned}
\frac{\partial}{\partial t} A^{P}(t, T) & =-a \theta^{Q} B^{P}-\frac{1}{2} \sigma^{2} B^{P 2}-\kappa c^{Q} C^{P} \\
\frac{\partial}{\partial t} C^{P}(t, T) & =\kappa C^{P}-\left[\psi^{Q}\left(B^{P}, C^{P} \delta^{Q}\right)-1\right]
\end{aligned}
$$

with the terminal conditions $A^{P}(T, T)=0, C^{P}(T, T)=0$, and

$$
B^{P}(t, T)=\frac{1}{a}\left(e^{-a(T-t)}-1\right) .
$$

Functions $A^{P}, B^{P}$, and $C^{P}$ are also needed in the next section to define a forward risk-neutral measure. The dynamics of bond prices depends on the random measure of the jump process, denoted $L^{Q}(d t, d z)$. This random measure is such that

$$
L_{t}^{Q}=\int_{0}^{\infty} \int_{-\infty}^{\infty} L^{Q}(d t, d z)
$$

and its expectation is $\mathbb{E}^{Q}\left(L^{Q}(d t, d z) \mid \mathcal{F}_{t}\right)=\lambda_{t}^{Q} \nu^{Q}(z) d z d t$. The next corollary presents the infinitesimal dynamics of bond prices. 
Corollary 3.5. Bond prices $P\left(t, T, r_{t}, \lambda_{t}^{Q}, I_{t}^{Q}\right)$ are ruled by the $S D E$

$$
\begin{aligned}
d P= & P r_{t} d t+B^{P}(t, T) P \sigma d W_{t}^{Q} \\
& +P \int_{-\infty}^{+\infty} \exp \left(\left(B^{P}(t, T), C^{P}(t, T) \delta^{Q}\right) z\right)-1 L^{Q}(d t, d z) \\
& -P \lambda_{t}^{Q}\left[\psi^{Q}\left(B^{P}(t, T), C^{P}(t, T) \delta^{Q}\right)-1\right] d t,
\end{aligned}
$$

where $L^{Q}(d t, d z)$ is the random measure of the jump process.

Proof. According to the Itô lemma for semi-martingales, $P\left(t, T, r_{t}, \lambda_{t}^{Q}, I_{t}^{Q}\right)$ is such that

$$
\begin{aligned}
& d P=P_{t}+\kappa\left(c^{Q}-\lambda_{t}^{Q}\right) P_{\lambda} d t+a\left(\theta^{Q}-r_{t}\right) P_{r} d t \\
& +\frac{1}{2} \sigma^{2} P_{r r} d t+P_{r} \sigma d W_{t}^{Q} \\
& +\int_{-\infty}^{+\infty} P\left(t, T, r_{t}+z, \lambda_{t}^{Q}+\delta^{Q}|z|, I_{t}^{Q}+(|z|, 1)^{\top}\right)-P L^{Q}(d t, d z),
\end{aligned}
$$

where partial derivatives are obtained from Eqs. (31) and (32).

From the last corollary, we infer that the instantaneous growth rate for the bond price is well equal to the short-term rate, $\mathbb{E}\left(\frac{d P}{P} \mid \mathcal{F}_{t}\right)=r_{t} d t$, as the sum of all other terms in Eq. (33) is a martingale.

Note that the function $\theta^{Q}(t)$ fitting an observed yield curve is approached in practice by a staircase function matching modeled and observed bond prices, denoted $P^{o b s}\left(0, t_{i}\right)$ in this paragraph, for a given set of maturities $\left(t_{1}, t_{2}, \ldots t_{n}\right)$. Construction of $\theta^{Q}(t)$ requires calculation of $B^{P}\left(0, t_{i}\right)$ and $C^{P}\left(0, t_{i}\right)$ (as defined in Corollary 3.4) for all maturities considered. Next, the values of $A^{P, o b s}\left(0, t_{i}\right)$ fitting observed prices are obtained using the relation

$$
A^{P, o b s}\left(0, t_{i}\right)=\log \left(P^{o b s}\left(0, t_{i}\right)\right)-B^{P}\left(0, t_{i}\right) r_{0}-C^{P}\left(0, t_{i}\right) \lambda_{0}^{Q} \quad t_{i} \in\left(t_{1}, t_{2}, \ldots t_{n}\right) .
$$

According to Eq. (32), $\theta^{Q}(s)$ is a function satisfying the following relations for all maturities:

$$
\begin{aligned}
& A^{P, o b s}\left(0, t_{i}\right)-A^{P, o b s}\left(0, t_{i-1}\right)= \\
& \quad-a \int_{t_{i-1}}^{t_{i}} \theta^{Q}(s) B^{P}\left(s, t_{i}\right) d s-\frac{1}{2} \sigma^{2} \int_{t_{i-1}}^{t_{i}} B^{P}\left(s, t_{i}\right)^{2} d s-\kappa c^{Q} \int_{t_{i}-1}^{t_{i}} C^{P}\left(s, t_{i}\right) d s,
\end{aligned}
$$

assuming that $\theta^{Q}(s)=\theta^{Q}(i)$ is constant over the interval $\left[t_{i-1} t_{i}\right)$, which leads to the following staircase approximation:

$$
\begin{aligned}
\theta^{Q}(i) \approx & -\frac{1}{a \Delta_{i}} \frac{A^{P, o b s}\left(0, t_{i}\right)-A^{P, o b s}\left(0, t_{i-1}\right)}{\left(B^{P}\left(s, t_{i}\right)-B^{P}\left(s, t_{i-1}\right)\right)}-\frac{1}{2} \frac{\sigma^{2}}{a}\left(B^{P}\left(s, t_{i}\right)+B^{P}\left(s, t_{i-1}\right)\right) \\
& -\frac{\kappa}{a} c^{Q} \frac{\left(C^{P}\left(s, t_{i}\right)-C^{P}\left(s, t_{i-1}\right)\right)}{\left(B^{P}\left(s, t_{i}\right)-B^{P}\left(s, t_{i-1}\right)\right)} .
\end{aligned}
$$

If the model is used for options pricing, the current yield curve has to be accurately reproduced to avoid model arbitrage. If the model is used to conduct an econometric analysis, it is better to set $\theta(t)$ to a constant value, as the purpose is to explain the dynamics of the term structure rather than to perfectly fit it. 


\section{Pricing of options}

This section illustrates how our model can be used for the pricing of interest rate derivatives under a forward measure. The yield for maturity $T-S$ at time $T$ is denoted by $Y(T, S)$ and is defined as

$$
\begin{aligned}
Y(T, S) & :=-\frac{1}{S-T} \log P(T, S) \\
& =-\frac{A^{P}(T, S)}{S-T}-\frac{B^{P}(T, S)}{S-T} r_{T}-\frac{C^{P}(T, S)}{S-T} \lambda_{T}^{Q} .
\end{aligned}
$$

The payoff paid at time $S \geq T$ by an European option written on $Y(T, S)$ is denoted by $V(Y(T, S))$. Examples of such instruments are caplets $(V(Y(T, S))=N(S-T)[Y(T, S)-$ $\left.k]_{+}\right)$, floorlets $\left(V(Y(T, S))=N(S-T) N[k-Y(T, S)]_{+}\right.$, and options for zero-coupon bonds $\left(V(Y(T, S))=N[\exp (-Y(T, S)(S-T))-k]_{+}\right)$, where $N$ is the principal and $k$ is the strike. The option price is the expectation for this discounted payoff under the risk-neutral measure:

$$
f\left(t, r_{t}, \lambda_{t}\right)=\mathbb{E}^{Q}\left(e^{-\int_{t}^{S} r_{s} d s} V(Y(T, S)) \mid \mathcal{F}_{t}\right)
$$

As recommended by Brigo and Mercurio (2007), it is better to evaluate the last expression under the $S$-forward measure. This avoids numerical inaccuracies related to the approximation of $\exp \left(-\int_{t}^{S} r_{s} d s\right)$ because the discount factor is drawn from Eq. (35) under the forward measure. If the market admits at least one risk-neutral measure $Q$, an equivalent probability measure to $Q$ is defined using the change in numeraire technique. The $S$-forward measure has as numeraire the zero-coupon bond of maturity $S$. Under this measure, the price of any financial assets, divided by the numeraire $P(t, S)$, is a martingale and the price of the derivative is

$$
\begin{aligned}
\mathbb{E}^{Q}\left(e^{-\int_{t}^{S} r_{s} d s} V(Y(T, S)) \mid \mathcal{F}_{t}\right) & =P(t, S) \mathbb{E}^{S}\left(V(Y(T, S)) \mid \mathcal{F}_{t}\right) \\
& =P(t, S) \int_{0}^{+\infty} V(y) f_{Y(T, S)}(y) d y
\end{aligned}
$$

where $f_{Y(T, S)}(y)$ is the density of $Y(T, S)$ under the forward measure. If the market value of a cash account is $B_{t}=e^{\int_{0}^{t} r_{s} d s}$, the Radon-Nykodym derivative defining the $S$-forward measure is

$$
\frac{d F^{S}}{d Q}=\frac{1}{B_{S}} \frac{B_{0}}{P(0, S)}=\left(e^{\int_{0}^{S} r_{s} d s} \mathbb{E}^{Q}\left(e^{-\int_{0}^{S} r_{s} d s} \mid \mathcal{F}_{0}\right)\right)^{-1}
$$

To calculate the expected payoff under $F^{S}$, the easiest approach is to approximate the pdf for $Y(T, S)$ using a discrete Fourier transform (DFT). To perform such a calculation, the Laplace function for the yield is needed.

Corollary 3.6. The Laplace transform of $Y(T, S)$ at time $t \leq T$ under the forward measure $F^{S}$, denoted by $\varphi^{t, S}($.$) , is given by$

$$
\begin{aligned}
& \varphi^{t, S}\left(w, r_{t}, \lambda_{t}\right)=\mathbb{E}^{S}\left(e^{w Y(T, S)} \mid \mathcal{F}_{t}\right)= \\
& \quad \exp \left(A^{F}(t, T)-A^{P}(t, S)+\left(B^{F}(t, T)-B^{P}(t, S)\right) r_{t}+\left(C^{F}(t, T)-B^{P}(t, S)\right) \lambda_{t}^{Q}\right),
\end{aligned}
$$

where $A^{P}(t, S), B^{P}(t, S), A^{P}(t, S)$ are defined in the corollary 3.4 for a zero coupon bond of maturity $S$. And where $A^{F}(t, T), B^{F}(t, T)$ and $C^{F}(t, T)$ are solutions of a system of ODEs:

$$
\left\{\begin{array}{l}
\frac{\partial}{\partial t} A^{F}(t, T)=-a \theta^{Q}(t) B^{F}-\kappa c^{Q} C^{F}-\frac{1}{2} \sigma^{2} B^{F 2} \\
\frac{\partial}{\partial t} B^{F}(t, T)=a B^{F}+1 \\
\frac{\partial}{\partial t} C^{F}(t, T)=\kappa C^{F}-\left[\psi^{Q}\left(B^{F}, C^{F} \delta^{Q}\right)-1\right]
\end{array}\right.
$$


and satisfy the terminal conditions:

$$
\begin{aligned}
& A^{F}(T, T)=\left(1-\frac{w}{S-T}\right) A^{P}(T, S), \\
& B^{F}(T, T)=\left(1-\frac{w}{S-T}\right) B^{P}(T, S), \\
& C^{F}(T, T)=\left(1-\frac{w}{S-T}\right) C^{P}(T, S) .
\end{aligned}
$$

Proof. By definition of the forward measure and using the fact that $\mathcal{F}_{t} \subset \mathcal{F}_{T}$, the Laplace transform of $Y(T, S)$ is given by

$$
\begin{aligned}
\mathbb{E}^{S}\left(e^{w Y(T, S)} \mid \mathcal{F}_{t}\right) & =\frac{\mathbb{E}^{Q}\left(\left(e^{\int_{0}^{S} r_{s} d s} \mathbb{E}^{Q}\left(e^{-\int_{0}^{S} r_{s} d s} \mid \mathcal{F}_{0}\right)\right)^{-1} e^{w Y(T, S)} \mid \mathcal{F}_{t}\right)}{e^{-\int_{0}^{t} r_{s} d s} \mathbb{E}^{Q}\left(e^{-\int_{t}^{S} r_{s} d s} \mid \mathcal{F}_{t}\right)\left(\mathbb{E}^{Q}\left(e^{-\int_{0}^{S} r_{s} d s} \mid \mathcal{F}_{0}\right)\right)^{-1}} \\
& =\frac{\mathbb{E}^{Q}\left(e^{-\int_{t}^{T} r_{s} d s} \mathbb{E}^{Q}\left(e^{-\int_{T}^{S} r_{s} d s+w Y(T, S)} \mid \mathcal{F}_{T}\right) \mid \mathcal{F}_{t}\right)}{\mathbb{E}^{Q}\left(e^{-\int_{t}^{S} r_{s} d s} \mid \mathcal{F}_{t}\right)} .
\end{aligned}
$$

The $\mathcal{F}_{T}$ conditional expectation in this equation is

$$
\mathbb{E}^{Q}\left(e^{-\int_{T}^{S} r_{s} d s+w Y(T, S)} \mid \mathcal{F}_{T}\right)=e^{w Y(T, S)} \mathbb{E}^{Q}\left(e^{-\int_{T}^{S} r_{s} d s} \mid \mathcal{F}_{T}\right) .
$$

According to Corollary 3.4, the two terms in this product are such that

$\mathbb{E}^{Q}\left(e^{-\int_{T}^{S} r_{s} d s+w Y(T, S)} \mid \mathcal{F}_{T}\right)=\exp \left(\left(1-\frac{w}{S-T}\right)\left(A^{P}(T, S)+B^{P}(T, S) r_{T}+C^{P}(T, S) \lambda_{T}^{Q}\right)\right)$.

Applying Proposition (2.2) allows us to complete the proof.

The next result introduces the discretization framework to build the density of $Y(T, S)$, under the forward measure. Note that it is possible to use the same algorithm to approach the distribution of $r_{t}$ under the real and risk-neutral measures.

Proposition 3.7. Let $M$ be the number of steps used in the DFT and let $\Delta_{y}=\frac{2 y_{\max }}{M-1}$ be the discretization step. We denote $\Delta_{z}=\frac{2 \pi}{M \Delta_{y}}$ and

$$
z_{j}=(j-1) \Delta_{z}
$$

for $j=1 \ldots M$. The values of $f_{Y(T, S)}($.$) at points y_{k}=-\frac{M}{2} \Delta_{y}+(k-1) \Delta_{y}$ are approached by the sum

$$
f_{Y(T, S)}\left(y_{k}\right) \approx \frac{2}{M \Delta_{y}} \operatorname{Re}\left(\sum_{j=1}^{M} \delta_{j} \varphi^{t, S}\left(i z_{j}, r_{t}, \lambda_{t}\right)(-1)^{j-1} e^{-i \frac{2 \pi}{M}(j-1)(k-1)}\right),
$$

where $\delta_{j}=\frac{1}{2} 1_{\{j=1\}}+1_{\{j \neq 1\}}$.

Proof. The density of $Y(T, S)$ is retrieved by calculating the Fourier transform of $\varphi^{t, T}(i z)$ as

$$
\begin{aligned}
f_{Y(T, S)}\left(y_{k}\right) & =\frac{1}{2 \pi} \mathcal{F}\left[\varphi^{t, S}\left(i z, r_{t}, \lambda_{t}\right)\right](y) \\
& =\frac{1}{2 \pi} \int_{-\infty}^{+\infty} \varphi^{t, S}\left(i z, r_{t}, \lambda_{t}\right) e^{-i y_{k} z} d z \\
& =\frac{1}{\pi} \operatorname{Re}\left(\int_{0}^{+\infty} \varphi^{t, S}\left(i z, r_{t}, \lambda_{t}\right) e^{-i y_{k} z} d z\right),
\end{aligned}
$$


where the last equality comes from the fact that $\varphi^{t, S}\left(z, r_{t}, \lambda_{t}\right)$ and $\varphi^{t, T}\left(-z, r_{t}, \lambda_{t}\right)$ are complex conjugate. At points $y_{k}=-\frac{M}{2} \Delta_{y}+(k-1) \Delta_{y}$, the last integral is approached with the trapezoid rule

$$
\int_{a}^{b} h(z) d z=\frac{h(a)+h(b)}{2} \Delta_{z}+\sum_{k=1}^{M-1} h\left(a+k \Delta_{z}\right) \Delta_{z}
$$

and leads to the following estimate for $f_{Y(T, S)}\left(y_{k}\right)$ :

$$
\begin{aligned}
f_{Y(T, S)}\left(y_{k}\right) & \approx \frac{1}{\pi} \operatorname{Re}\left(\sum_{j=1}^{M} \delta_{j} \varphi^{t, S}\left(i z_{j}, r_{t}, \lambda_{t}\right) e^{-i y_{k} z_{j}} \Delta_{z}\right) \\
& \approx \frac{1}{\pi} \operatorname{Re}\left(\sum_{j=1}^{M} \delta_{j} \varphi^{t, S}\left(i z_{j}, r_{t}, \lambda_{t}\right)(-1)^{j-1} e^{-i \frac{2 \pi}{M}(j-1)(k-1)} \Delta_{z}\right)
\end{aligned}
$$

Once the density of $Y(T, S)$ is obtained using the DFT, the option price is approached by a weighted sum of payoffs:

$$
\mathbb{E}^{Q}\left(e^{-\int_{t}^{T} r_{s} d s} V(Y(T, S)) \mid \mathcal{F}_{t}\right)=P(t, T) \sum_{k=1}^{M+1} V\left(y_{k}\right) f_{Y(T, S)}\left(y_{k}\right) \Delta_{y} .
$$

The feasibility of this method is illustrated for caplets in the next section.

\section{Econometric calibration and numerical applications}

Fitting the model to a real time series would allow us to demonstrate the need to include a self-exciting jump process in short-term rate models. However, the calibration is not direct because jumps and their arrival intensity cannot be directly observed. Furthermore, the density of interest rates does not admit any closed-form solution. It is thus not possible to infer parameters via direct $\log$ likelihood maximization. However, several alternatives exist. Ait-Sahalia et al. (2014a) used a GMM approach to measure contagion in stocks markets. Ait-Sahalia et al. (2014b) used an alternative method based on matching modeled and market prices for 5-year CDS quotes. Chen and Poon (2013) combined moments and price matching for variance swaps. At high frequency, jumps can be detected and fitted separately using the asymptotic properties of Brownian motion, as illustrated by Mancini (2009). Still at high frequency, Barndorff-Nielsen and Shephard $(2004,2006)$ defined and used bipower and multipower variation processes to estimate jumps.

As we work at low frequency (daily data observed over ten years), we instead opt for a peakover-threshold procedure similar to the one proposed by Embrechts et al. (2011). This method is simple and robust. Consider a discrete record $\left\{r_{t_{0}}, r_{t_{1}}, \ldots, r_{t_{n}}\right\}$ of $n+1$ observations of $r_{t}$, equally spaced at $t_{j}=j h$ for a given lag $h$. The variations in interest rates are denoted by $\Delta r_{i}=r_{t_{i}}-r_{t_{i-1}}$. The unobservable number of jumps observed in these intervals is denoted by $\Delta_{i} N=N_{t_{i}}-N_{t_{i-1}}$. The threshold $g(\alpha)$ is a deterministic function of the lag between the observations and a threshold parameter $\alpha \in] 0,1]$ (the method for determining $\alpha$ is explained later). It is assumed that when the drift-adjusted $\Delta r_{i}$ is greater than $g(\alpha)$, it is likely that some jumps occurred. To determine $g(\alpha)$ and the drift of $\Delta r_{i}$, we first use log likelihood maximization to fit a mean-reverting process without jumps, also called a Vasicek model: $\Delta r_{i} \sim a^{g}\left(\theta^{g}-r_{t_{i-1}}\right) h+\sigma^{g} W_{h}$. 
The Vasicek model also serves as a benchmark for evaluating our model. $g(\alpha)$ is defined as the $\alpha$-percentile of $\sigma^{g} W_{h}: g(\alpha)=\sigma^{g} \sqrt{h} \Phi^{-1}(\alpha)$. Under the assumption that when a jump occurs the Brownian part is nonsignificant compared to the jump, the dynamics of the short-term rate is approached by

$$
\left\{\begin{array}{rlrl}
\Delta r_{i} & \sim a^{g}\left(\theta^{g}-r_{t_{i-1}}\right) h+\sigma^{g} W_{h}, \Delta_{i} N=0 & & \left|\Delta r_{i}-a^{g}\left(\theta^{g}-r_{t_{i-1}}\right) h\right| \leq g(\alpha) \\
\Delta r_{i} \sim a^{g}\left(\theta^{g}-r_{t_{i-1}}\right) h+J, \Delta_{i} N=1 & & \left|\Delta r_{i}-a^{g}\left(\theta^{g}-r_{t_{i-1}}\right) h\right|>g(\alpha)
\end{array} .\right.
$$

Once the jumps are filtered, the Brownian process, the jump distribution, and the intensity process are fitted separately via log likelihood maximization. If we still assume that the mean reversion level $\theta(t)$ is constant, the following three optimization problems are solved numerically to find an estimate of the parameters:

$$
\left\{\begin{array}{l}
(a, \theta, \sigma)=\arg \max \sum_{i=1}^{n} \log \text { Normal Pdf }\left(\Delta r_{i}, a\left(\theta-r_{t_{i-1}}\right) h, \sigma \sqrt{h}\right) 1_{\text {\{no jumpat } \left._{i}\right\}} \\
\left(\rho^{+}, \rho^{-}, p\right)=\arg \max \sum_{i=1}^{N_{t_{n}}} \log \text { Double Expo Pdf }\left(J_{i}, \rho^{+}, \rho^{-}, p\right) \\
\left(\kappa, c, \delta, \lambda_{0}\right)=\arg \max \sum_{i=1}^{n} \log \text { Poisson Pdf }\left(\Delta_{i} N, \lambda_{i} h\right),
\end{array}\right.
$$

where the jump arrival intensity is discretized as : $\lambda_{i}=\lambda_{i-1}+\kappa\left(c-\lambda_{i-1}\right) h+\delta J 1_{\left\{\text {jumpat }_{i}\right\}}$ for $i=1$ to $n$. Because jumps smaller (in absolute value) than the threshold $g(\alpha)$ are censored, we have a more accurate fit if we replace the double-exponential pdf by its censored pdf, as described in Appendix B.

This procedure was applied to EONIA time series data from 1 January 2004 to 31 December 2014. The index is a weighted average of all overnight unsecured lending transactions (in euros) between prime banks, and is representative of short-term rates. Its evolution is plotted in Figure 3. From January 2004 to December 2005, EONIA values oscillated around $2.08 \%$ and then continuously increased to reach $4.3 \%$ in September 2007. From this date to 15 September 2008, the day on which Lehman Brothers collapsed, the EONIA value remained at this level but the volatility was higher than in previous periods. From the end of 2008 to July 2009, EONIA values followed a downward trend as a result of European Central Bank (ECB) measures to improve global liquidity. In the first few weeks of 2010, anxiety about excessive European national debts again increased interest rates and volatility. In mid-2012, owing to successful fiscal consolidation and implementation of structural reforms in the countries most at risk and various policy measures taken by the ECB (such as quantitative easing), financial stability in the Eurozone significantly improved and interest rates steadily decreased to reach a floor at approximately 0.13\% in July 2012. A peak in activity observed from December 2013 to June 2014 is linked to renewed fears about the solvency of Greece.

Table 3 presents estimates of $a^{g}, \theta^{g}$, and $\sigma^{g}$ for the Vasicek model used to define the threshold. The first graph in Figure 1 presents a QQ plot of the residuals $\left(\sigma^{g}\right)^{-1}\left(\Delta r_{i}-a^{g}\left(\theta^{g}-r_{t_{i-1}}\right) h\right)$ versus a normal distribution. As expected, the quality of the fit is poor because, in contrast to the Vasicek model, the EONIA is not stationary. Choice of the threshold parameter $\alpha$ is problematic and has an impact on the number of filtered jumps. To determine its best value, we compute the Jarque-Bera statistic for residuals $\sigma^{-1}\left(\Delta r_{i}-a\left(\theta-r_{t_{i-1}}\right) h\right)$ on days when no jump is observed for $\alpha$ ranging from $50 \%$ to $90 \%$. The results, summarized in Tables 1 and 2, reveal that the residuals are normal for $\alpha=56 \%$, with an $85 \%$ asymptotic $p$-value (skewness close to zero and kurtosis nearly equal to three). This is confirmed by the third and fourth QQ plots in Figure 1.

For a such value of the threshold parameter, $36 \%$ of observations are considered as jumps and the average amplitude of positive and negative jumps is approximately ten basis points (bps). The cause of these jumps is not clearly identified. However, we can reasonably hypothesize that 
they are related to a sudden change in the overall credit exposure of reference banks selected for the EONIA. Alternatively, they are the consequence of changes in the monetary policy of European central banks, such as adjustments of the overnight rate, or quantitative easing.

The EONIA variations separately attributed to Brownian and jump terms are plotted in Figure 2. It is evident that diffusion causes only small oscillations located in a [-1 bps $+1 \mathrm{bps}]$, whereas the jump process explains the movements of highest amplitude. The clustering of jumps is clearly visible. We can observe that EONIA variations attributed to Brownian motion are equal to $\pm 0.01 \%$ from 1 January 2004 to 31 August 2007. This is because the EONIA was reported to only two digits during this period. Since 1 September 2007, the EONIA has been reported to three digits.

Table 3 lists the drift and Brownian volatility parameter for the short-term rate when $\alpha=56 \%$. Once jumps are removed from the sample, the volatility of the Brownian component decreases from $1.51 \%$ to $0.09 \%$. The mean reversion speed decreases from 0.52 to 0.36 and the mean reversion level $\theta$ changes from $1.16 \%$ to $0.85 \%$.

Table 4 shows fitted parameters for double-exponential jumps when $\alpha=56 \%$. In absolute value, parameters $\rho^{+}$and $\rho^{-}$are very close. The probability is also slightly lower for observation of an upward jump than for a downward jump $(p=46 \%)$. The fourth and fifth graphs of Figure 1 show QQ plots of filtered jumps versus a double-exponential distribution. These confirm that choosing this distribution provides a reasonable fit for a threshold parameter of $56 \%$.

Table 5 presents the calibration results for the intensity process. The mean reversion speed for $\lambda_{t}$ is high at 5.77. The mean reversion level is stable at 59.50 jumps per year. The parameter that tunes the self-excitation of $r_{t}$ is $\delta$ and the calibration reveals that $\delta$ is significantly not null. This confirms the presence of clustering effects in the EONIA dynamics. The value of $\delta$ is high because EONIA shocks are small on average: a jump of 5 bps causes an increase in intensity of 1.80 .

In Figure 3, the first two graphs show simulated sample paths for $r_{t}$ and $\lambda_{t}$. The simulated path for $r_{t}$ oscillates more than the real one, but contains periods of decline, sharp increases, and stability that are comparable to the EONIA trend. The simulated sample path for $\lambda_{t}$, similar to the real one, remains in the interval [90-210]. Without perfectly matching the EONIA time series, these graphs reveal that the model shares some common features with EONIA trends. Figure 4 compares daily EONIA variations simulated using the Hawkes-diffusion model and the Vasicek model to real variations. The results reveal that in contrast to the Vasicek model, the presence of a self-exciting jump process in the interest rate dynamics generates nonstationary increments and more realistic sample paths.

In contrast to one-factor models, which cannot simulate nonparallel shocks for the yield curve, our model can generate a wide range of deformations. This point is illustrated in the first graph of Figure 5, which shows selected humped and inverse humped yield curves. The initial yield curve is the one computed with parameters obtained via econometric calibration. The sensitivity of the curve to changes in $p, \rho^{+}, \rho^{-}$, and $\delta$ is illustrated in the three next graphs of Figure 5 . Reducing the probability of upward jumps, $p$, flattens the yield curve and drives down expected jumps. Increasing $\rho^{+}$lowers the average size of positive jumps, equal to $\frac{1}{\rho^{+}}$. This decreases prospective EONIA growth and flattens the yield curve. Reducing the parameter that tunes the clustering effect, $\delta$, is equivalent to decreasing the overall frequency of jumps. As these jumps are slightly negative on average, this increases future short-term rates and the whole yield curve is steeper. 
The econometric calibration relies on historical data, and parameters fitted using such an approach define the dynamics of $r_{t}$ under the real measure of probability $P$. To estimate the value of parameters under the risk-neutral measure, we work with market data for zero-coupon yields (in euro) bootstrapped from swap curves observed over the last decade (13 maturities ranging from 1 to 20 years). The risk premiums $\xi$ and $\gamma$ defining an equivalent risk-neutral measure according to Eq. (19) are inferred on a given day by minimizing the sum of spreads between model-based and swap yields. In practice, these premiums are not constant over time and are directly related to the level of risk aversion in financial markets. Figure 6 shows the evolution of these parameters fitted at a regular interval of 5 days of trading. $\xi$ is negative and slightly increasing from 2004 to 2011. As $\theta^{Q}=\theta-\frac{\xi \sigma}{a}$, the mean reversion level during this period is greater the one under the real measure $P$, and greater than the EONIA trend. This explains the upward slope of swap curves. After 2011, $\theta^{Q}$ and $\theta$ tend to converge as $\xi$ decreases. The plot of EONIA versus $\theta^{Q}$ in Figure 6 reveals that markets always anticipate reversion of short-term rates towards higher levels. The decrease in the spread between $r_{t}$ and $\theta^{Q}$ from 2011 to 2014 explains the flattening observed for swap curves.

It is also evident that $\gamma$ oscillated around -20 during the last 4 years. Around the credit crunch period, $\gamma$ was significantly higher. The last graph of Figure 6 presents the parameters $\rho^{+}$and $\rho^{-}$defining the size of jumps. Both parameters decreased in absolute value from 2006 to 2011. As they are inversely proportional to the average jump amplitude, this means that the size of jumps and indirectly the overall volatility of $r_{t}$ evaluated by the market increased in this period.

Figure 7 shows selected implied volatility curves for a set of 1-year caplets with a 1-year tenor. Prices were obtained using a Fourier transform with $M=2^{10}$ discretization steps and $y_{\max }=$ 0.10. Implied volatility values were obtained by inverting the Black-Scholes formula for caplets. These graphs illustrate the sensitivity of implied volatility to a change in key parameters defining the jump process. The parameters and risk premiums are those retrieved for 31 December 2014 via econometric calibration. The probability $p$ of observing a positive jump has a large impact on caplet prices and volatility. When $p$ increases, jumps are more often positive than negative on average. Then the likelihood that the yield will hit the strike increases, as does the implied volatility. A change in the frequency $\lambda_{t}$ changes the steepness of the implied volatility curve. An increase in $\delta$ indirectly increases the number of jumps that are negative on average. Then the probability that the short-term rate will hit the strike at maturity is lower and the implied volatility decreases. Finally, changes in $\rho^{+}$or $\rho^{-}$also cause a clear parallel shift of implied volatility.

\section{Conclusions}

We proposed a model for interest rates with a feedback mechanism that reproduces clustering effects. The approach consists of adding a self-exciting jump process, also called a Hawkes process, to classical mean-reverting Brownian dynamics. In contrast to similar credit risk models in which jumps are unidirectional or even constant, shocks in our approach are positive or negative. Regardless of their direction, shocks increase the intensity of jump arrivals. In addition to the propagation feature, the model has several advantages over existing methods. First, this is a two-factor model that replicates a wide range of yield curves, including humped, decreasing, and increasing curves. Second, it belongs to the affine model class and semi-closed-form expressions are available for its moment-generating function and for bond prices. There also exists a class of risk neutral measures under which the dynamics of the short-term rate is similar to the dynamics under the real measure. Third, the model is easy to calibrate using the peak-over-threshold procedure, which is robust and easy to implement for econometric purposes. Finally, such a model can also be used to price most European interest rate derivatives under a forward measure via 
Fourier transform.

Our study of the EONIA over the last ten years provides clear evidence of the presence of clustering effects. This confirms the need to introduce a propagation mechanism in the dynamics of short-term rates. Moreover, combining econometric calibration with an analysis of past swap curves allows us to filter risk premiums and the evolution of parameters under the market measure. Two trends emerge from this exercise. First, the mean reversion level rate towards which the EONIA revert decreases continuously and converges to spot rates. This explains the flattening of the swap curve observed since 2010. Second, the average amplitude of shocks decreases, which reduces the overall volatility of rates under the pricing measure.

\section{Appendix A}

Proposition 5.1. If $J_{i}$ is a double-exponential random variable such as that defined by Eq. (1), the moment-generating function of a weighted sum of $J_{i}$ and $\left|J_{i}\right|$ is equal to

$$
\mathbb{E}\left(e^{z_{1} J_{i}+z_{2}\left|J_{i}\right|}\right)=p \frac{\rho^{+}}{\rho^{+}-\left(z_{1}+z_{2}\right)}+(1-p) \frac{\rho^{-}}{\rho^{-}-\left(z_{1}-z_{2}\right)}
$$

if $\left(z_{1}+z_{2}\right)<\rho^{+},\left(z_{1}-z_{2}\right)>\rho^{-}$.

Proof. By construction, the moment-generating function is equal to the sum

$$
\mathbb{E}\left(e^{z_{1} J_{i}+z_{2}\left|J_{i}\right|}\right):=p \mathbb{E}\left(e^{\left(z_{1}+z_{2}\right) J_{i}} \mid J_{i} \geq 0\right)+(1-p) \mathbb{E}\left(e^{\left(z_{1}-z_{2}\right) J_{i}} \mid J_{i} \leq 0\right) .
$$

To evaluate conditional expectations in the last equation, we need the conditional densities. As the conditional probabilities are

$$
\begin{aligned}
& P\left(J_{i} \leq x \mid J_{i} \geq 0\right)=\frac{P\left(0 \leq J_{i} \leq x\right)}{P\left(J_{i} \geq 0\right)}=\left(1-e^{-\rho^{+} x}\right), \\
& P\left(J_{i} \leq x \mid J_{i} \leq 0\right)=\frac{P\left(J_{i} \leq x\right)}{P\left(J_{i} \leq 0\right)}=e^{-\rho^{-} x}
\end{aligned}
$$

the conditional densities are

$$
\begin{aligned}
& \frac{d}{d x} P\left(J_{i} \leq x \mid J_{i} \geq 0\right)=\rho^{+} e^{-\rho^{+} x}, \\
& \frac{d}{d x} P\left(J_{i} \leq x \mid J_{i} \leq 0\right)=-\rho^{-} e^{-\rho^{-} x} .
\end{aligned}
$$

On the basis of these results, the conditional expectations in Eq. (38) are given by the following expressions:

$$
\begin{aligned}
\mathbb{E}\left(e^{\left(z_{1}-z_{2}\right) J_{i}} \mid J_{i} \leq 0\right) & =\int_{-\infty}^{0}-e^{\left(z_{1}-z_{2}\right) x} \rho^{-} e^{-\rho^{-} x} d x \\
& =\frac{\rho^{-}}{\rho^{-}-\left(z_{1}-z_{2}\right)} \text { if }\left(z_{1}-z_{2}\right)>\rho^{-},
\end{aligned}
$$

and

$$
\begin{aligned}
\mathbb{E}\left(e^{\left(z_{1}+z_{2}\right) J_{i}} \mid J_{i} \geq 0\right) & =\int_{0}^{+\infty} e^{\left(z_{1}+z_{2}\right) x} \rho^{+} e^{-\rho^{+} x} d x \\
& =\frac{\rho^{+}}{\rho^{+}-\left(z_{1}+z_{2}\right)} \quad \text { if }\left(z_{1}+z_{2}\right)<\rho^{+}
\end{aligned}
$$




\section{Appendix B}

Proposition 5.2. If $J_{i}$ is a double-exponential random variable as defined by Eq. (1) and is censored in the interval $[-g, g]$, its truncated pdf is

$$
\frac{d}{d x} P\left(J_{i} \leq x \mid J_{i} \geq g \cup J_{i} \leq-g\right)= \begin{cases}+\frac{p}{p e^{-\rho^{+} g}+(1-p) e^{\rho^{-} g}} \rho^{+} e^{-\rho^{+} x} & x \geq g \\ 0 & -g<x<g . \\ -\frac{1-p}{p e^{-\rho^{+} g}+(1-p) e^{\rho^{-} g}} \rho^{-} e^{-\rho^{-} x} & x \leq-g\end{cases}
$$

Proof. The cumulative distribution function (cdf) for $J_{i}$ censored in the interval $[-g, g]$ can be developed as follows:

$$
\begin{aligned}
P\left(J_{i}\right. & \left.\leq x \mid J_{i} \geq g \cup J_{i} \leq-g\right)=\frac{P\left(J_{i} \leq x \cap\left(J_{i} \geq g \cup J_{i} \leq-g\right)\right)}{P\left(J_{i} \geq g \cup J_{i} \leq-g\right)} \\
& =\frac{P\left(J_{i} \leq x \cap J_{i} \geq g\right)+P\left(J_{i} \leq x \cap J_{i} \leq-g\right)}{P\left(J_{i} \geq g\right)+P\left(J_{i} \leq-g\right)} \\
& =\frac{P\left(J_{i} \geq g\right) P\left(J_{i} \leq x \mid J_{i} \geq g\right)+P\left(J_{i} \leq-g\right) P\left(J_{i} \leq x \mid J_{i} \leq-g\right)}{P\left(J_{i} \geq g\right)+P\left(J_{i} \leq-g\right)} .
\end{aligned}
$$

From Eq. (2) we infer that the denominator in this last expression is

$$
P\left(J_{i} \geq g\right)+P\left(J_{i} \leq-g\right)=p e^{-\rho^{+} g}+(1-p) e^{\rho^{-} g} .
$$

The conditional probabilities in the numerator are given by

$$
\begin{aligned}
P\left(J_{i} \leq x \mid J_{i} \geq g\right) & =I_{x \geq g} \frac{P\left(g \leq J_{i} \leq x\right)}{P\left(J_{i} \geq g\right)} \\
& =I_{x \geq g}\left(1-e^{-\rho^{+}(x-g)}\right)
\end{aligned}
$$

and

$$
\begin{aligned}
P\left(J_{i} \leq x \mid J_{i} \leq-g\right) & =I_{x \leq-g} \frac{P\left(J_{i} \leq x\right)}{P\left(J_{i} \leq-g\right)}+I_{x>-g} \\
& =I_{x \leq-g} e^{-\rho^{-}(x+g)}+I_{x>-g}
\end{aligned}
$$

If we insert these expressions into Eq. (41), we have the following result for the truncated cdf of $J_{i}$ :

$$
\begin{aligned}
P\left(J_{i} \leq x \mid J_{i} \geq g \cup J_{i} \leq-g\right)= & \frac{p}{p e^{-\rho^{+} g}+(1-p) e^{\rho^{-} g}} I_{x \geq g}\left(e^{-\rho^{+} g}-e^{-\rho^{+} x}\right) \\
& +\frac{1-p}{p e^{-\rho^{+} g}+(1-p) e^{\rho^{-} g}} I_{x \leq-g} e^{-\rho^{-} x} \\
& +\frac{1-p}{p e^{-\rho^{+} g}+(1-p) e^{\rho^{-} g}} I_{x>-g} e^{\rho^{-} g}
\end{aligned}
$$

and we can conclude by deriving this cdf.

\section{References}

[1] Ait-Sahalia, Y., Cacho-Diaz, J., Laeven, R.J.A., Modeling financial contagion using mutually exciting jump processes. To appear in J. of Fin. Econ. 2014 (a)

[2] Ait-Sahalia Y., Laeven R.J.A, Pelizzon L., Mutual excitation in Eurozone sovereign CDS. To appear in J. of Fin. Econ. 2014 (b) 
[3] Bacry E., Delattre S.,Hoffmann M., Muzy J.F., Modelling microstructure noise with mutually exciting point processes. Quant. Finance 2013, 13(1), 65-77

[4] Bauwens, L., Hautsch, N., Handbook of financial time series: modelling financial high frequency data using point processes. Springer, Berlin, 2009.

[5] Barndorff-Nielsen, O. E., Shephard, N., Power and bipower variation with stochastic volatility and jumps (with discussion). J. of Fin. Econ. 2004, 2, 1-48.

[6] Barndorff-Nielsen, O. E., Shephard, N., Econometrics of testing for jumps in financial economics using bipower variation. J. of Fin. Econ. 2006, 4, 1-30

[7] Brigo D. and Mercurio F., Interest rate models - Theory and Practice. Springer Finance, 2007.

[8] Chavez-Demoulin, V., Davison, A.C., McNeil, A.J., A point process approach to value-atrisk estimation. Quant. Finance 2005, 5 (2), 227-234.

[9] Chavez-Demoulin, V., McGill J.A., High-frequency financial data modeling using Hawkes processes. J. of Bank. and Fin. 2012, 36, 3415-3426

[10] Chen K., and Poon S.-H., Variance swap premium under stochastic volatility and selfexciting jumps. SSRN work. pap. 2013, SSRN-id2200172.

[11] Cox J.C., Ingersoll J.E, Ross S.A., A theory of the term structure of interest rates, Econometrica 1985, 53, 385-407.

[12] Dai Q.,Singleton K.J., Specification analysis of affine term structure models. J. of Fin. 2000, 55, 1943-1978.

[13] Dassios A. ,Jang J., A double shot noise process and its application in insurance. J of Math. and Syst. Sci. 2012, 2, 82-93

[14] Duffie D., Kan R., A yield-factor model of interest rates. Math. Fin. 1996, 6, 379-406.

[15] Eberlein E., Kluge W., Exact pricing formulae for caps and swaptions in a Levy term structure model. J. of Comput. Fin. 2006, 9 (2), 99-125.

[16] Embrechts, P., Liniger, T., Lu, L., Multivariate Hawkes processes: an application to financial data. J. of Applied Proba. 2011, 48 (A), 367-378.

[17] Errais, E., Giesecke, K., Goldberg, L.R., Affine point processes and portfolio credit risk. SIAM J. of Fin. Math. 2010, 1, 642-665.

[18] Filipović D., Tappe S., Existence of L?vy term structure models Fin. and Stoch. 2008, 12 (1) $83-115$.

[19] Giot, P., Market risk models for intraday data. Euro. J. of Fin. 2005, 11 (4), 309-324.

[20] Hainaut D., MacGilchrist R. An Interest rate tree driven by a L?vy process. J. of Deriv. 2010, 18 (2), 33-45.

[21] Hawkes, A., Point sprectra of some mutually exciting point processes. J. of the Royal Stat. Soc. B 1971 (a), 33, 438-443.

[22] Hawkes, A., Spectra of some self-exciting and mutually exciting point processes. Biometrika 1971 (b), 58, 83-90. 
[23] Hawkes A. and Oakes D., A cluster representation of a self-exciting process. J. of Applied Proba. 1974, 11, 493-503.

[24] Hull. J. White. A., Pricing interest rate derivatives. Rev. of Fin. Stud. 1990, Vol 3(4), $573-592$

[25] Mancini C. , Non-parametric threshold estimation for models with stochastic diffusion coefficient and jumps. Scand. J. of Stat. 2009, 36, 270-296.

[26] Salmon M. Tham W.W., Time deformation and term structure of interest rates. SSRN work. pap. 2007 , SSRN-id999841.

[27] Vasicek O., "An equilibrium characterization of the term structure." J. of Fin. Econ. 1977, $5,177-188$.

[28] Zhang X.W. , Glynn P.W., Giesecke K., Rare event simulation for a generalized Hawkes process. Proceedings of the 2009 Winter Simulation Conference. 


\begin{tabular}{ccccc}
\hline \hline $\begin{array}{c}\text { Threshold } \\
\alpha\end{array}$ & $\begin{array}{c}\text { Jarque-Bera } \\
\text { statistic }\end{array}$ & $\begin{array}{c}\text { Asymptotic } \\
p \text {-value }\end{array}$ & Skewness & Kurtosis \\
\hline \hline $55 \%$ & 0.87 & $64.79 \%$ & -0.05 & 2.95 \\
$\mathbf{5 6 \%}$ & $\mathbf{0 . 3 1}$ & $\mathbf{8 5 . 5 5 \%}$ & $\mathbf{- 0 . 0 3}$ & $\mathbf{3 . 0 1}$ \\
$57 \%$ & 2.79 & $24.79 \%$ & -0.09 & 3.07 \\
$58 \%$ & 13.36 & $0.13 \%$ & -0.14 & 3.30 \\
$59 \%$ & 20.25 & $0.00 \%$ & -0.15 & 3.38 \\
$60 \%$ & 39.74 & $0.00 \%$ & -0.14 & 3.62 \\
$70 \%$ & 510.43 & $0.00 \%$ & -0.17 & 5.27 \\
$80 \%$ & 1484.73 & $0.00 \%$ & -0.28 & 6.76 \\
$90 \%$ & 2618.16 & $0.00 \%$ & -0.41 & 7.86 \\
\hline \hline
\end{tabular}

Table 1: Jarque-Bera statistic and $p$-value for residuals (jumps excluded) filtered using the peaks-over-threshold procedure for different threshold parameters. For $\alpha=56 \%$ the skewness is close to 0 and the kurtosis is equal to 3 .

\begin{tabular}{cccc}
\hline \hline $\begin{array}{c}\text { Threshold } \\
\alpha\end{array}$ & $\begin{array}{c}\text { Yearly frequency } \\
\text { of jumps }\end{array}$ & $\mathbb{E}\left(\max \left(J_{i}, 0\right)\right)$ & $\left|\mathbb{E}\left(\min \left(J_{i}, 0\right)\right)\right|$ \\
\hline \hline $55 \%$ & 100.98 & $0.095 \%$ & $0.086 \%$ \\
$\mathbf{5 6 \%}$ & $\mathbf{9 2 . 8 5}$ & $\mathbf{0 . 1 0 3 \%}$ & $\mathbf{0 . 0 9 1 \%}$ \\
$57 \%$ & 83.37 & $0.116 \%$ & $0.098 \%$ \\
$58 \%$ & 76.23 & $0.124 \%$ & $0.107 \%$ \\
$59 \%$ & 72.47 & $0.128 \%$ & $0.112 \%$ \\
$60 \%$ & 67.38 & $0.137 \%$ & $0.118 \%$ \\
$70 \%$ & 44.50 & $0.187 \%$ & $0.163 \%$ \\
$80 \%$ & 31.81 & $0.229 \%$ & $0.208 \%$ \\
$90 \%$ & 21.00 & $0.285 \%$ & $0.275 \%$ \\
\hline \hline
\end{tabular}

Table 2: Descriptive statistics for jumps filtered using the peaks-over-threshold procedure for different threshold parameters.

\begin{tabular}{cccccc}
\hline \hline & All data & Std Err. & & $\alpha=56 \%$ & Std Err. \\
\hline \hline$a^{g}$ & 0.5200 & $0.0026[\mathrm{~A} 3]$ & $a$ & 0.3603 & 0.0016 \\
$\theta^{g}$ & $1.1621 \%$ & $0.0106 \%$ & $\theta$ & $0.85 \%$ & $0.0112 \%$ \\
$\sigma^{g}$ & $1.5135 \%$ & $0.0302 \%$ & $\sigma$ & $0.09 \%$ & $0.0502 \%$ \\
\hline Log likelihood & 15618.44 & (2820 observations) & & 14767.70 & $(1781$ observations $)$ \\
\hline \hline
\end{tabular}

Table 3: The first column of this table shows the parameters of the Vasicek model, fitted by log likelihood maximization. The second columns presents the volatility, the level and speed of mean reversion, after removing variations of interest rates considered as jumps. 


\begin{tabular}{ccc}
\hline \hline & $\alpha=56 \%$ & Std. Err. \\
\hline \hline$\rho^{+}$ & 969.21 & 1.40 \\
$\rho^{-}$ & -1093.58 & 1.44 \\
$p$ & $0.46[\mathrm{~A} 4]$ & 0.00 \\
\hline $\mathbb{E}\left(J_{i}\right)$ & $-0.0029 \%$ & \\
\hline Log likelihood & 6575.89 & \\
\hline \hline
\end{tabular}

Table 4: Fitted parameters for the distribution of jumps.

\begin{tabular}{ccc}
\hline \hline & $\alpha=56 \%$ & Std. Err. \\
\hline \hline$\lambda_{0}$ & 102.64 & 0.71 \\
$\kappa$ & 5.77 & 0.01 \\
$\delta$ & 3613.89 & 4.19 \\
$c$ & 59.50 & 0.07 \\
\hline Log likelihood & -1586.66 & \\
\hline \hline
\end{tabular}

Table 5: Parameters defining the dynamics of the intensity of $N_{t}$. 

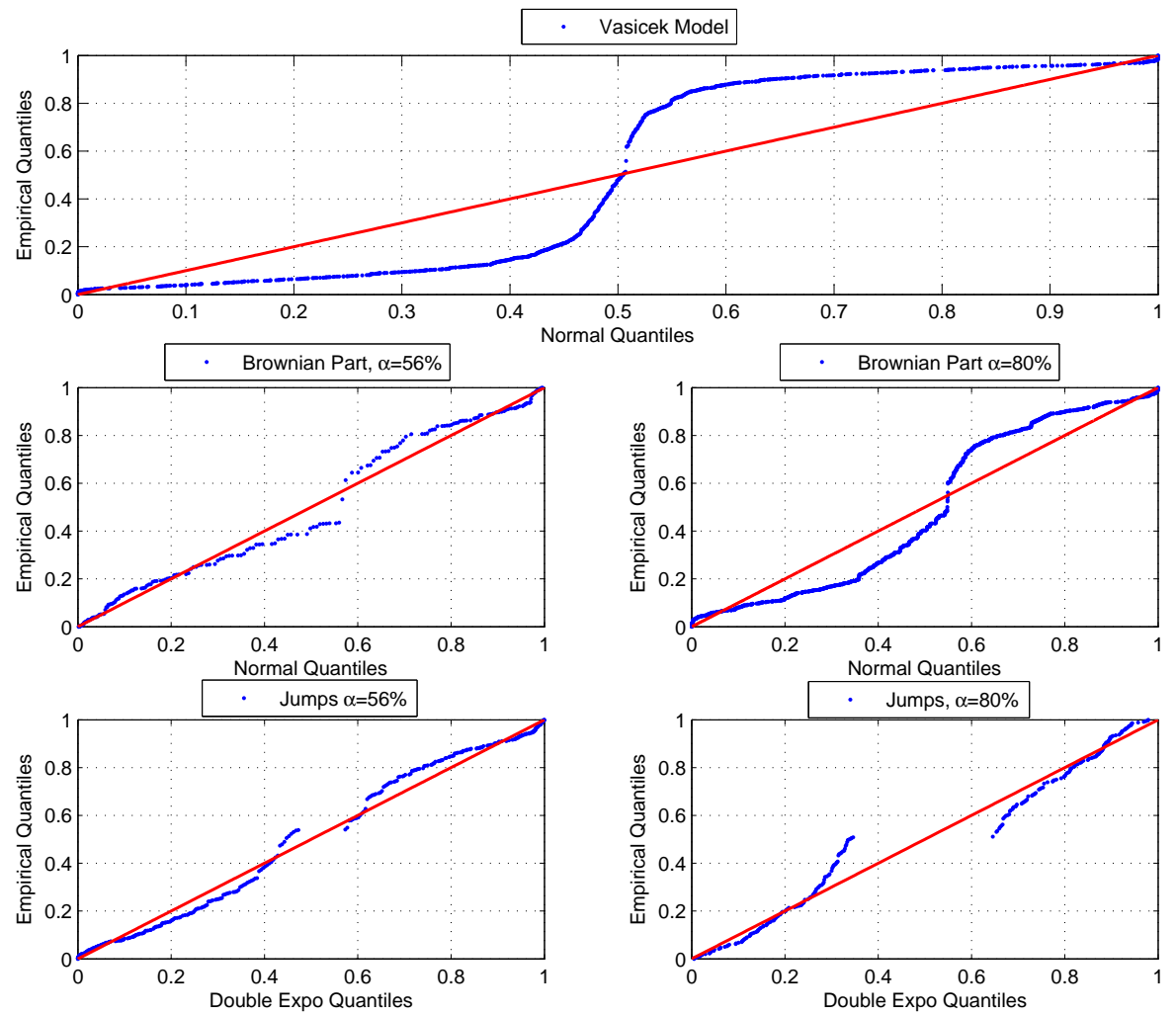

Figure 1: The first graph is a QQ plot of residuals $\left(\sigma^{g}\right)^{-1}\left(\Delta r_{i}-a^{g}\left(\theta^{g}-r_{t_{i-1}}\right) h\right)$ for the Vasicek model versus a normal distribution. The second and third graphs are QQ plots of residuals $\sigma^{-1}\left(\Delta r_{i}-a\left(\theta-r_{t_{i-1}}\right) h\right)$ versus a normal distribution when jumps are removed from the data set for $\alpha=56 \%$ and $\alpha=80 \%$. The last two graphs are QQ plots of filtered jumps versus a double-exponential distribution for $\alpha=56 \%$ and $\alpha=80 \%$. 

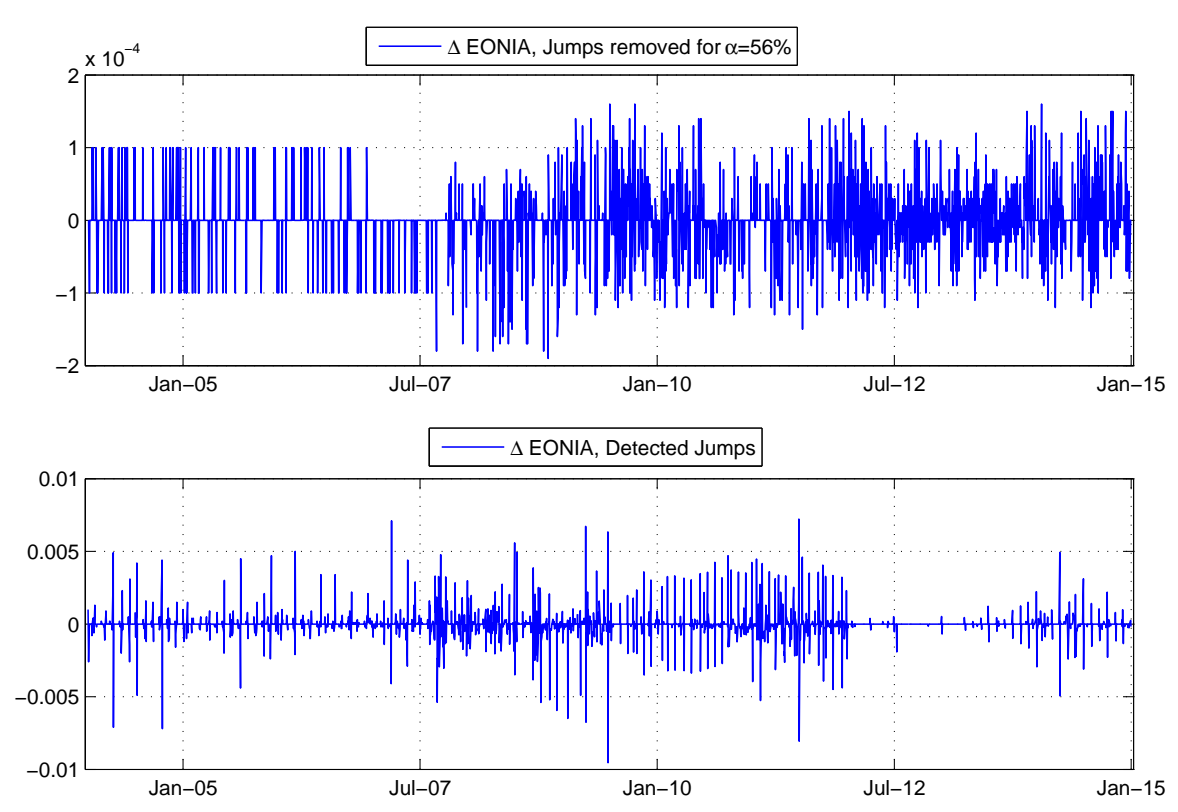

Figure 2: The first graph shows EONIA variations attributed to the diffusion part of $r_{t}$. The second graph shows EONIA variations considered as jumps. Both series are filtered with a threshold parameter set to $56 \%$. EONIA variations attributed to Brownian motion are equal to $\pm 0.01 \%$ from $1 / 1 / 2004$ to $31 / 8 / 2007$ because the EONIA was only reported to two digits over this period. Since $1 / 9 / 2007$, the EONIA has been reported to three digits. 

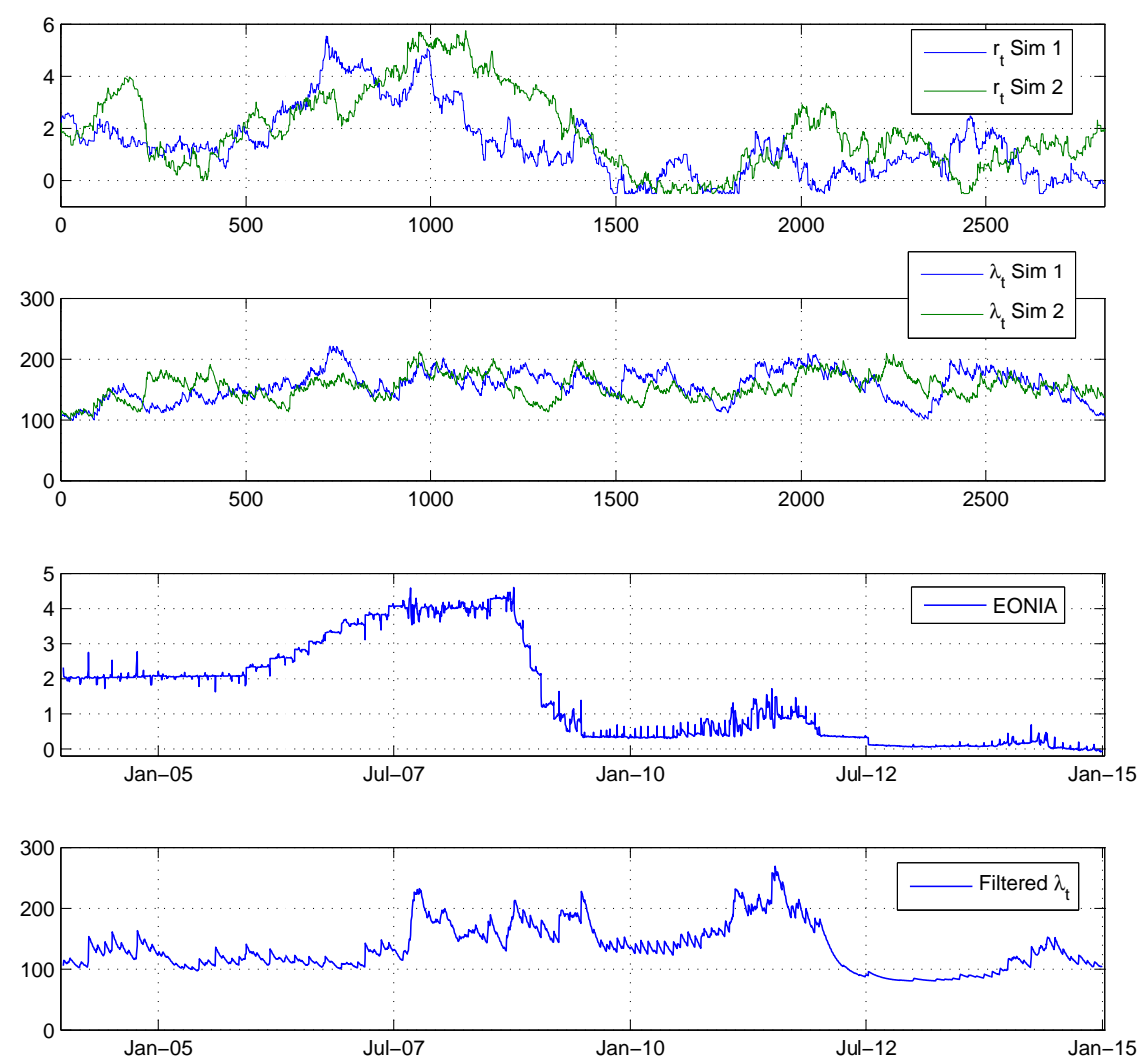

Figure 3: The first two graphs show simulated sample paths for $r_{t}$ and $\lambda_{t}$. The last two graphs show the daily evolution for EONIA and the filtered intensity $\lambda_{t}$, respectively, from $1 / 1 / 2004$ to $31 / 12 / 2014$.
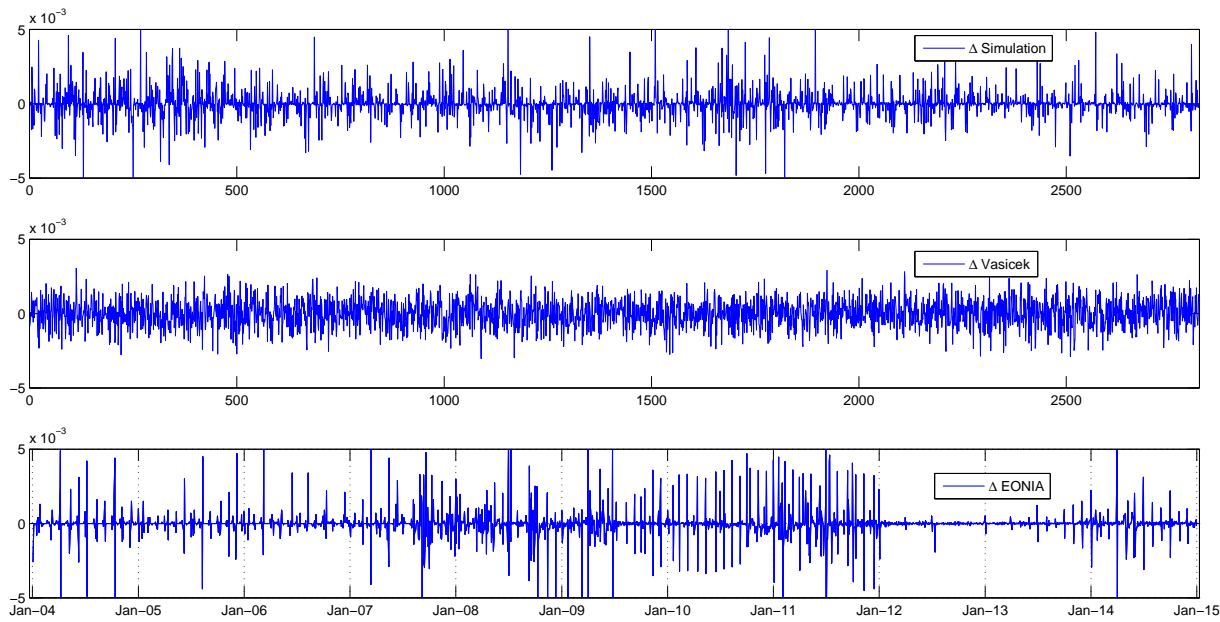

Figure 4: The first graph shows daily variations in the interest rate, $\Delta r_{t}$, simulated using parameters obtained for $\alpha=56 \%$. The second graph shows daily variations in $\Delta r_{t}$ simulated using a Vasicek model fitted to the same data set. The last graph shows EONIA variations observed over the period 2004-2015. 

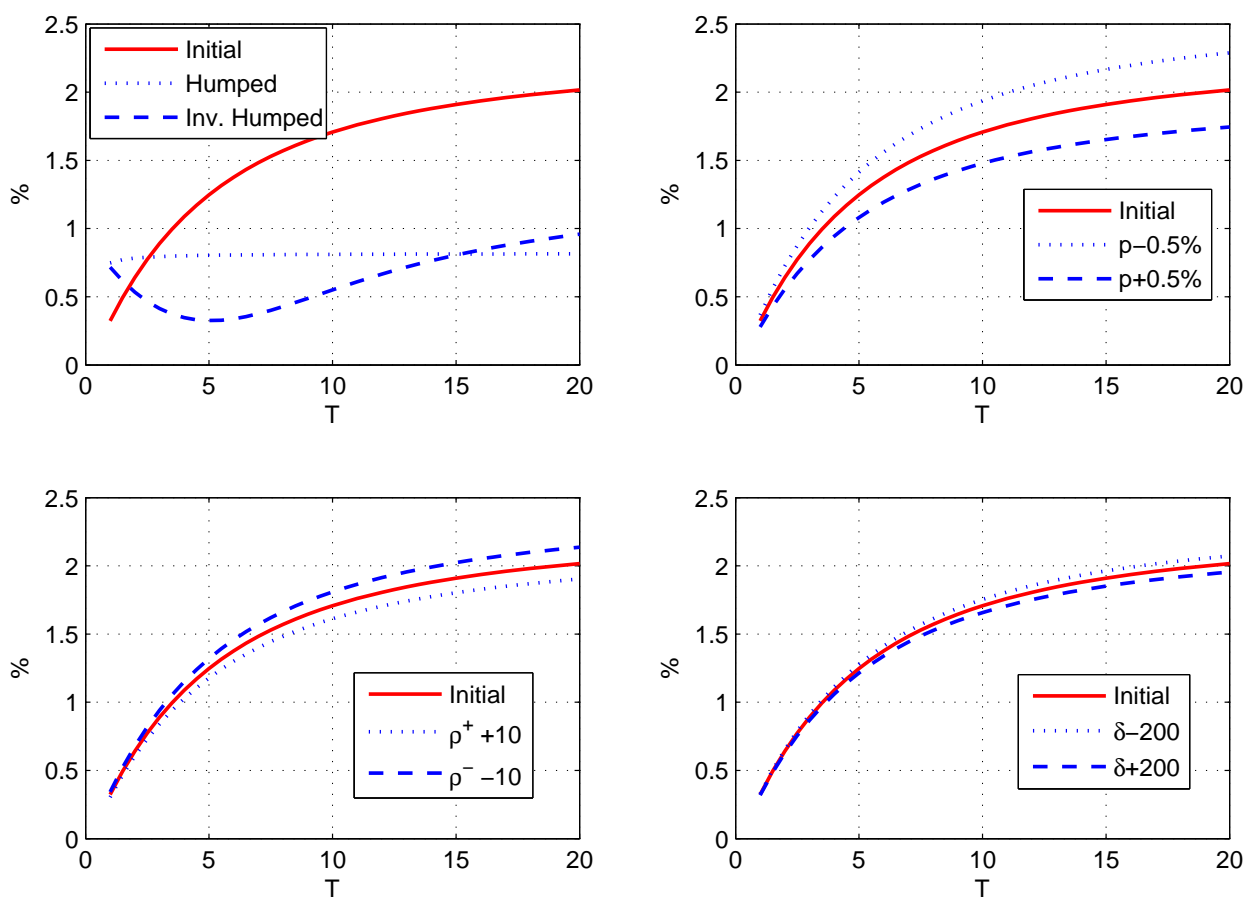

Figure 5: The first graph shows selected (model-based) yield curves. The three other graphs illustrate the sensitivity of the yield curve to changes in key parameters defining the jump process. The initial parameters are those obtained via econometric calibration. 

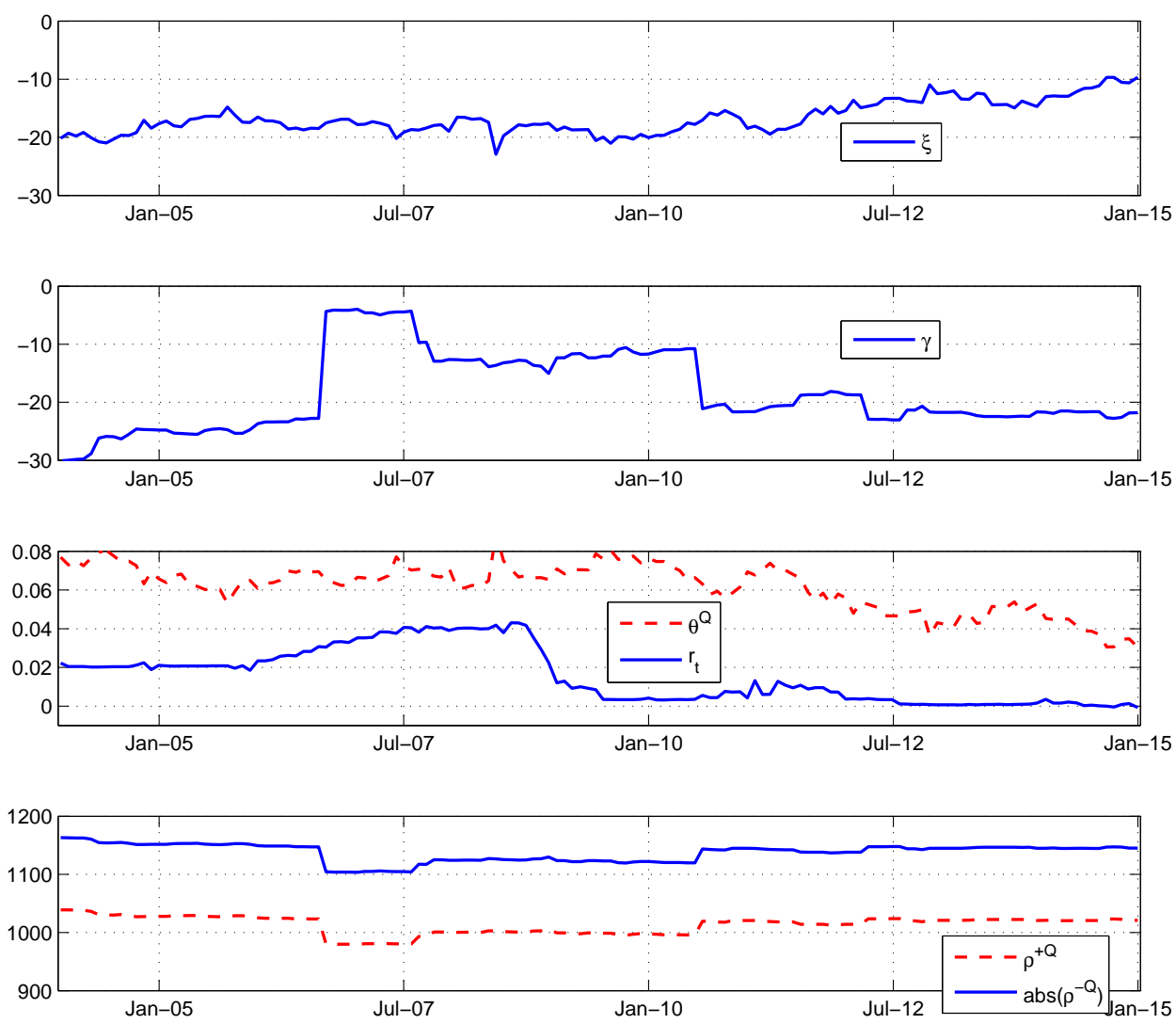

Figure 6: The first two graphs present the risk premiums $\xi$ and $\gamma$ minimizing the spread in the yield to maturity between observed and modeled data. The third figure compares the EONIA with the mean reversion parameter under $Q$. The last graph shows the evolution of the jump parameters $\rho^{+Q}$ and $\rho^{-Q}$. Parameters under $P$ are fitted for a confidence level of $\alpha=56 \%$. 

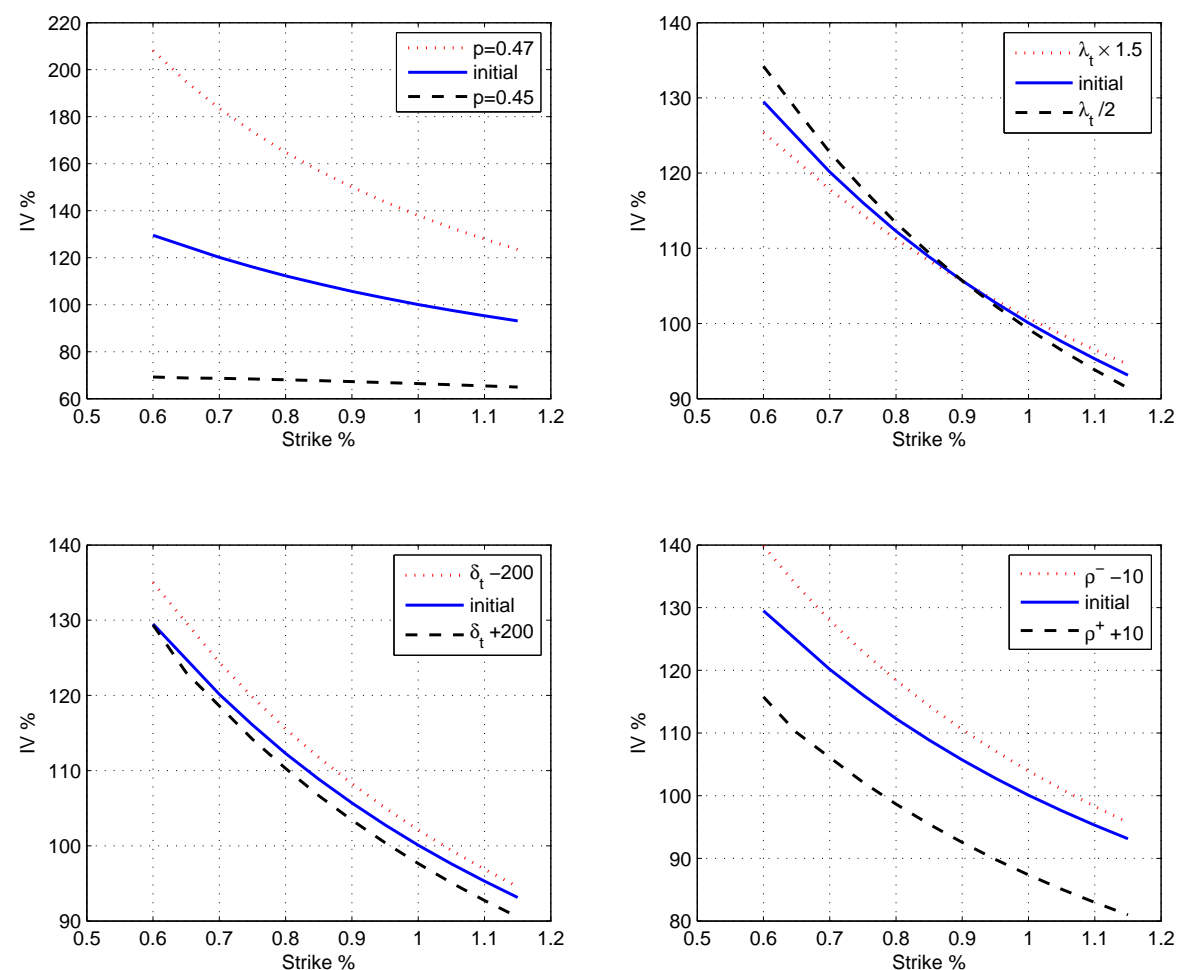

Figure 7: Graphs showing the sensitivity of caplet implied volatility to changes in key parameters determining the jump process. The caplets all have maturity and tenor of 1 year. 